\title{
ON OBJECTIVE SURFACE RATES
}

BY

\author{
H. STUMPF AND J. BADUR
}

Ruhr-Universität Bochum, Bochum, Germany

\begin{abstract}
In this paper we derive objective, in the sense of surface, rates of tensors and we give a correct formulation of a two-dimensional continuum. Furthermore we present objective rates of tensors on the boundary line enclosing the surface under consideration. It can be considered as a first step to derive objective rates for generalized continua as Cosserat continua and Kirchhoff-Love type nonlinear shell theories.
\end{abstract}

1. Introduction. Objective time derivatives were introduced into the constitutive equations primarily to describe properly the time change of stress and strain tensors. Starting from the pioneering papers of Zaremba [36] and Jaumann [12], the concept of objective rates has been the subject of several works. Among them the fruitful concept of "convective derivatives" was given by Oldroyd [22]. It can be considered as a method of time differentiation of any tensor field attached to the deformed body, but performed after some mapping into a place where a comparison of different tensors can be made. Since Oldroyd has used the gradient of deformation for the mapping operation, all objective derivatives based on the deformation gradient or its parts are called "convective rates." Special cases are presently known as ZarembaJaumann, Cotter-Rivlin [4] or Rivlin-Ericksen derivatives related to strain rates, and the Truesdell derivative [31] related to stress rates. Additional arguments in favor of the convective derivatives were given by Sedov [25] and Masur [17]. The latter has shown how the Zaremba-Jaumann derivative is connected with that of Oldroyd.

Recently, great efforts have been made for a correct formulation of two-dimensional thermomechanics of a curved surface. For many phenomena, like dynamics of a phase interfaces layer [34], viscoelastic 2-dimensional flow of the products of friction and wear [37], capillary flow [9], bubble dynamics [11], dynamics of a crack [27], elastoviscoplasticity of thin shells, etc., it is required to define useful constitutive equations. Frequently, the constitutive equations of differential or rate type are used, since they are easier to implement in computer codes than equations of integral type. These phenomenological relations should contain information about the surface geometry as well as objective surface rates both of stresses and strains.

The aim of this paper is to consider a correct formulation of the two-dimensional continuum and to derive objective, but in the sense of surface, rates of tensors. 
Our considerations are restricted to convective time derivatives inside of a surface. Furthermore we present objective rates of tensors on the boundary line enclosing the surface under consideration. It seems to us that this is a first step towards deriving objective rates for generalized continua as Cosserat continuum, Kirchhoff-Love type shell theories a. s. o.

2. Geometry of deformation. To analyze convective time derivatives we first need a unique description of a process of surface deformation. It will be sufficient to consider only initial and actual configurations of a smooth material surface bounded by a closed and smooth boundary curve (Fig. 1). According to the notation of Rational Mechanics introduced by Truesdell [31] we use capital letters of the Greek or Latin alphabet to describe the mechanical state of the initial surface and small letters for the actual one $[33, \S 178]$.

We assume that the motion of a two-dimensional material continuum is completely described by fields that are parametrized by two Gauss coordinate parameters and the parameter of time. Let the Lagrangian observer use the coordinate system $\left\{\Theta^{\Sigma}, T\right\}, \Sigma, \Omega, \Phi=1,2$, and the Eulerian observer the coordinate system $\left\{\theta^{\sigma}, t\right\}$, $\sigma, \omega, \varphi=1,2$. The Lagrangian $(T)$ and Eulerian $(t)$ time coordinates represent, of course, the same physical parameter, what leads to $T_{, t}=t_{, T}=1$ during an exchange of the observer.

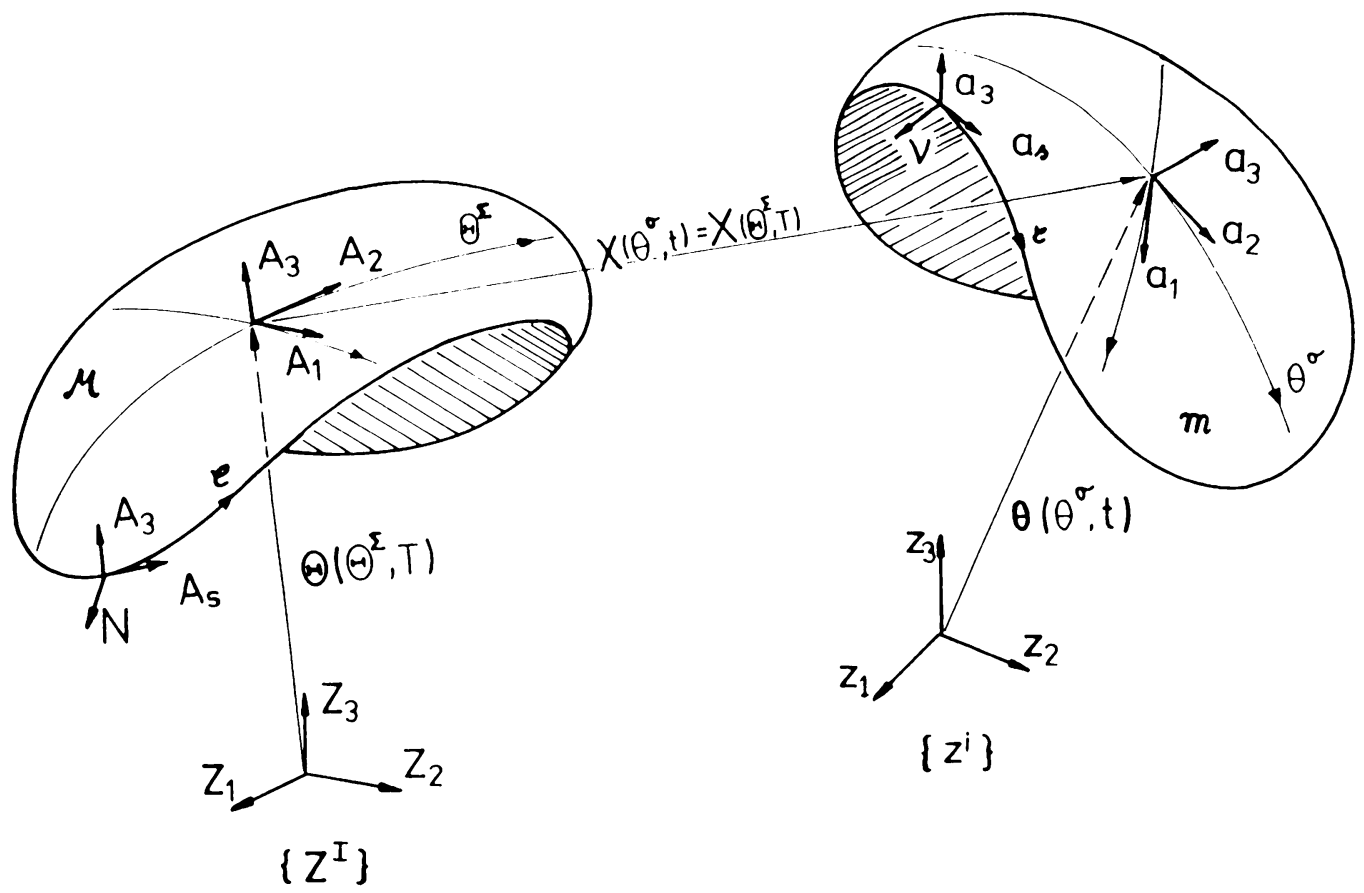

FIG. 1 . 
It is a matter of pure differential geometry to describe an internal as well as external property of a two-dimensional Riemannian continuum $m$ embedded into the threedimensional Euclidean space. Let $\boldsymbol{\theta}\left(\theta^{\sigma}, t\right)$ be its position vector and

$$
\mathbf{a}_{\sigma}(\theta, t)=\left(\partial / \partial \theta^{\sigma}\right) \boldsymbol{\theta}=\boldsymbol{\theta}_{, \sigma}=\left(z^{i}\left(\theta^{\varphi}, t\right) \mathbf{z}_{i}\right)_{, \sigma}=z_{, \sigma^{i}}^{i} \mathbf{z}_{i}
$$

the covariant base vectors. The orientation of $m$ is determined by the unit normal vector $\mathbf{a}_{3}=\mathbf{a}_{1} \times \mathbf{a}_{2} / a$. The basic notions of first and second fundamental form and related formulae can be recalled as [35]

$$
\begin{gathered}
a_{\sigma \omega}=\mathbf{a}_{\sigma} \cdot \mathbf{a}_{\omega}, \quad a^{\sigma \omega}=\mathbf{a}^{\sigma} \cdot \mathbf{a}^{\omega}, \quad a=\operatorname{det} a_{\sigma \omega}=a_{11} a_{22}-a_{12}^{2}, \quad \delta_{\sigma}^{\omega}=\mathbf{a}^{\omega} \cdot \mathbf{a}_{\sigma}, \\
b_{\sigma \omega}=\mathbf{a}_{\sigma, \omega} \cdot \mathbf{a}_{3}=-\mathbf{a}_{3, \sigma} \cdot \mathbf{a}_{\omega}, \quad h=\frac{1}{2} b_{\sigma}^{\sigma}, \quad h=\frac{b}{a}, \quad b=\operatorname{det} b_{\sigma \omega},
\end{gathered}
$$

where $h$ is half of the sum of the principal curvatures and is frequently called the "mean curvature" and $k$ is the product of the principal curvatures, generally called the "specific curvature."

Besides, the following Gauss-Weingarten relations hold:

$$
\begin{aligned}
\mathbf{a}_{\gamma, \sigma} & =\gamma_{\varphi \sigma}^{\omega} \mathbf{a}_{\omega}+b_{\varphi \sigma} \mathbf{a}_{3}, \\
\mathbf{a}_{, \sigma} & =-\gamma_{\omega \sigma}^{\varphi} \mathbf{a}^{\omega}+b_{\sigma}^{\varphi} \mathbf{a}_{3}, \\
\mathbf{a}_{3, \sigma} & =-b_{\sigma}^{\varphi} \mathbf{a}_{\varphi},
\end{aligned}
$$

where $\gamma_{\sigma \varphi}^{\omega}$ are the Christoffel coefficients. Other well-known relations will be mentioned as required in the course of the paper.

To define the 2-D gradient and divergence operators (Weatherburn [35]) we use the two-dimensional Hamilton operator $\nabla_{2}=\mathbf{a}^{\sigma} \partial_{\sigma}$;

$$
\begin{gathered}
\mathbf{a}=\boldsymbol{\theta} \otimes \nabla_{2} \equiv \gamma \operatorname{rad} \boldsymbol{\theta}=\mathbf{a}_{\varphi} \otimes \mathbf{a}^{\varphi}=a_{\sigma \varphi} \mathbf{a}^{\sigma} \otimes \mathbf{a}^{\varphi}, \\
\mathbf{b}=-\mathbf{a}_{3} \otimes \nabla_{2}=-\gamma \operatorname{rad} \mathbf{a}_{3}=b_{\sigma \varphi} \mathbf{a}^{\sigma} \otimes \mathbf{a}^{\varphi}, \\
\delta \operatorname{iv} \boldsymbol{\theta}=\boldsymbol{\theta} \cdot \nabla_{2}=2, \quad \delta \operatorname{iv} \mathbf{a}_{3}=-2 h, \quad \delta \operatorname{iv} \mathbf{a}=2 h \mathbf{a}_{3}, \\
\delta \operatorname{iv}\left(\gamma \operatorname{rad} \mathbf{a}_{3}\right)=-\left(4 h^{2}-2 h\right) \mathbf{a}_{3}-2 \gamma \operatorname{rad}(h) .
\end{gathered}
$$

Whereas $\mathbf{a}$ is the 2-D identity tensor $\left(\mathbf{a}=\mathbf{1}-\mathbf{a}_{3} \otimes \mathbf{a}_{3}\right)$, the symmetric tensor $\mathbf{b}$ is the internal measure of curvature. The Lagrangian form of the relations (2.3) referred to the surface $\mathscr{M}$ are

$$
\begin{aligned}
& \mathbf{A}\left(\Theta^{\Sigma}, T\right)=\Gamma \operatorname{rad} \boldsymbol{\Theta}=\mathbf{\Theta}_{, \Sigma} \otimes \mathbf{A}^{\Sigma}=\mathbf{A}_{\Sigma} \otimes \mathbf{A}^{\Sigma}=A_{\Omega \Sigma} \mathbf{A}^{\Omega} \otimes \mathbf{A}^{\Sigma}, \\
& \mathbf{B}\left(\Theta^{\Sigma}, T\right)=-\Gamma \operatorname{rad} \mathbf{A}_{3}=B_{\Omega \Sigma} \mathbf{A}^{\Omega} \otimes \mathbf{A}^{\Sigma}, \\
& \Delta \operatorname{iv} \boldsymbol{\Theta}=2, \quad \Delta \text { iv } \mathbf{A}_{3}=-2 \mathscr{H}, \quad \Delta \operatorname{iv} \mathbf{A}=2 \mathscr{H} \mathbf{A}_{3}, \\
& \Delta \operatorname{iv}\left(\Gamma \operatorname{rad} \mathbf{A}_{3}\right)=-\left(4 \mathscr{H}^{2}-2 \mathscr{K}\right) \mathbf{A}_{3}-2 \Gamma \operatorname{rad}(\mathscr{H}) .
\end{aligned}
$$

In contrary to the 3 -D continuum where the identity tensor $\mathbf{1}$ in the three-dimensional space cannot be distinguished for Lagrangian and Eulerian description, here the 2-D identity tensors $\mathbf{A}$ and a are different, but $\mathbf{1} \equiv \mathbf{A}+\mathbf{A}_{3} \otimes \mathbf{A}_{3} \equiv \mathbf{a}+\mathbf{a}_{3} \otimes \mathbf{a}_{3}$. Weatherburn's index free notation has been developed further by Gurtin and Murdoch [10], where additional results can be found. 
The complete information about the surface deformation $\mathscr{M} \rightarrow m$ can be given by the surface gradient of deformation defined as two-point tensor

$$
\mathbf{f}\left(\theta^{\sigma}, t, \Theta^{\Sigma}, T\right)=\Gamma \operatorname{rad} \boldsymbol{\theta}=\boldsymbol{\theta}, \Sigma \otimes \mathbf{A}^{\Sigma}=\left(z^{i}\left(\theta^{\sigma}\right) \mathbf{z}_{i}\right)_{, \Sigma} \otimes \mathbf{A}^{\Sigma}=\theta_{, \Sigma}^{\sigma} \mathbf{a}_{\sigma} \otimes \mathbf{A}^{\Sigma}
$$

and its inverse

$$
\mathbf{f}^{-1}\left(\theta^{\sigma}, t, \Theta^{\Sigma}, T\right)=\gamma \operatorname{rad} \boldsymbol{\Theta}=\boldsymbol{\Theta}_{, \sigma} \otimes \mathbf{a}^{\sigma}=\Theta_{, \sigma}^{\Sigma} \mathbf{A}_{\Sigma} \otimes \mathbf{a}^{\sigma} .
$$

The above definitions are in agreement with the general ansatz to describe the deformation via two-point tensors [33, §17]. However, if for any reason we prefer the Lagrangian or Eulerian one-point description, it may be performed through an appropriate use of the displacement vector $\chi\left(\theta^{\sigma}, t\right)=\mathbf{X}\left(\Theta^{\Sigma}, T\right)$ (Fig. 1). Then, a convective base $\mathbf{a}_{\Sigma}\left(\Theta^{\Omega}, T\right)=\boldsymbol{\Theta}_{, \Sigma}+\mathbf{X}_{, \Sigma}$ on the surface $m$ and a convective base $\mathbf{A}_{\sigma}\left(\theta^{\omega}, t\right)=\boldsymbol{\theta}_{, \sigma}+\chi_{, \sigma}$ on the surface $\mathscr{M}$ are induced. Also, the deformation gradient and its inverse become one-point measures

$$
\mathbf{f}\left(\Theta^{\Sigma}, T\right)=\mathbf{a}_{\Sigma} \otimes \mathbf{A}^{\Sigma}, \quad \mathbf{f}^{-1}\left(\theta^{\sigma}, t\right)=\mathbf{A}_{\sigma} \otimes \mathbf{a}^{\sigma},
$$

which will be discussed in detail in $§ 7.1$.

It is remarkable that for the Jacobian of deformation

$$
j=\operatorname{det} \mathbf{f}=\left(\frac{a}{A}\right)^{1 / 2} \operatorname{det}\left(\theta_{, \Sigma}^{\sigma}\right)>0
$$

and for the gradients of deformation (2.5) and (2.6), the 2-D analogies of the EulerNeumann formula $[31, \S 13]$ are valid:

$$
\Delta \operatorname{iv}\left(j \mathbf{f}^{-\mathrm{T}}\right)=0, \quad \delta \operatorname{iv}\left(j^{-1} \mathbf{f}^{\mathbf{T}}\right)=0,
$$

whereas $\mathbf{f f}^{-1}=\mathbf{a}, \mathbf{f}^{-1} \mathbf{f}=\mathbf{A}$.

In analogy to the 3-D proposition of Cauchy concerning the multiplicative decomposition of the deformation gradient into rotation and stretch, we postulate the polar decomposition

$$
\mathbf{f}=\vee \mathbf{r}=\mathbf{r} \cup,
$$

where $U=\sqrt{\mathbf{f}^{T} \mathbf{f}}, V=\sqrt{\mathbf{f f}^{T}}$ are understood as the 2-D stretch tensors and $\mathbf{r}$ as the 2-D orthogonal rotation tensor.

The first measure of deformation can be defined similarly to that in the Cauchy continuum as some kind of subtraction of the two matrices $\mathbf{a}$ and $\mathbf{A}$ after lifting to the same point:

$$
\begin{aligned}
\Gamma\left(\Theta^{\Phi}, T\right) & =\frac{1}{2}\left(\mathbf{f}^{\mathrm{T}} \mathbf{a f}-\mathbf{A}\right)=\frac{1}{2}\left(\theta_{, \Sigma}^{\sigma} \theta_{, \Omega}^{\omega} a_{\sigma \omega}-A_{\Sigma \Omega}\right) \mathbf{A}^{\Sigma} \otimes \mathbf{A}^{\Omega}, \\
\gamma\left(\theta^{\varphi}, t\right) & =\frac{1}{2}\left(\mathbf{a}-\mathbf{f}^{-\mathrm{T}} \mathbf{A} \mathbf{f}^{-1}\right)=\frac{1}{2}\left(a_{\sigma \omega}-\Theta_{, \sigma}^{\Sigma} \Theta_{, \omega}^{\Omega} A_{\Sigma \Omega}\right) \mathbf{a}^{\sigma} \otimes \mathbf{a}^{\omega} .
\end{aligned}
$$

As a result, the surface membrane strain tensors are obtained, where $\Gamma$ corresponds in 3-D continuum to the Green-St. Venant strain tensor and $\gamma$ to the Almansi-Hamel strain tensor $[31, \S 15]$.

The second possible surface deformation measures do not exist within the Euclidean space, because they describe the change of the internal property of the Riemannian surface. Thus, the symmetric surface tensors, able to describe such changes, 
can be the functions of curvature [26]

$$
\begin{aligned}
& \mathbf{K}\left(\Theta^{\Phi}, T\right)=-\left(\mathbf{f}^{\mathrm{T}} \mathbf{b f}-\mathbf{B}\right)=-\left(\theta_{, \Sigma}^{\sigma} \theta_{, \Omega}^{\omega} b_{\sigma \omega}-B_{\Sigma \Omega}\right) \mathbf{A}^{\Sigma} \otimes \mathbf{A}^{\Omega} \\
& \boldsymbol{\kappa}\left(\theta^{\varphi}, t\right)=-\left(\mathbf{b}-\mathbf{f}^{-\mathrm{T}} \mathbf{B} \mathbf{f}^{-1}\right)=-\left(b_{\sigma \omega}-\Theta_{,{ }_{\sigma}}^{\Sigma} \Theta_{, \omega}^{\Omega} B_{\Sigma \Omega}\right) \mathbf{a}^{\sigma} \otimes \mathbf{a}^{\omega}
\end{aligned}
$$

and bicurvature

$$
\begin{gathered}
\mathbf{M}\left(\Theta^{\Sigma}, T\right)=\frac{1}{2}\left(\mathbf{f}^{\mathbf{T}} \mathbf{b}^{2} \mathbf{f}-\mathbf{B}^{2}\right)=\mathbf{M}_{\Sigma \Omega} \mathbf{A}^{\Sigma} \otimes \mathbf{A}^{\Omega}, \\
\boldsymbol{\mu}\left(\theta^{\sigma}, t\right)=\frac{1}{2}\left(\mathbf{b}^{2}-\mathbf{f}^{-\mathrm{T}} \mathbf{B}^{2} \mathbf{f}^{-1}\right)=\mu_{\sigma \omega} \mathbf{a}^{\sigma} \otimes \mathbf{a}^{\omega} .
\end{gathered}
$$

As the 2-D analogue of Cauchy's dynamic postulate, we introduce a set of symmetric surface stress measures

$$
\mathbf{n}\left(\theta^{\varphi}, t\right)=n^{\sigma \omega} \mathbf{a}_{\sigma} \otimes \mathbf{a}_{\omega}, \quad \mathbf{m}\left(\theta^{\varphi}, t\right)=m^{\sigma \omega} \mathbf{a}_{\sigma} \otimes \mathbf{a}_{\omega}, \quad \mathbf{l}\left(\theta^{\varphi}, t\right)=l^{\sigma \omega} \mathbf{a}_{\sigma} \otimes \mathbf{a}_{\omega},
$$

the membrane stress, moment and bi-moment tensors, which are work-conjugate to the strain measures $\boldsymbol{\gamma}, \boldsymbol{\kappa}$, and $\boldsymbol{\mu}$. However, from the point of a Lagrangian observer three symmetric surface tensors of second Piola-Kirchhoff type can be postulated also [26]

$$
\begin{gathered}
\mathbf{N}\left(\Theta^{\Phi}, T\right)=N^{\Sigma \Omega} \mathbf{A}_{\Sigma} \otimes \mathbf{A}_{\Omega}, \quad \mathbf{M}\left(\Theta^{\Phi}, T\right)=M^{\Sigma \Omega} \mathbf{A}_{\Sigma} \otimes \mathbf{A}_{\Omega}, \\
\mathbf{L}\left(\Theta^{\Phi}, T\right)=L^{\Sigma \Omega} \mathbf{A}_{\Sigma} \otimes \mathbf{A}_{\Omega},
\end{gathered}
$$

naturally work-conjugated to the strain measures $\boldsymbol{\Gamma}, \mathbf{K}, \mathbf{M}$. If follows from (2.13) that $\boldsymbol{\mu}$ as well as $\mathrm{M}$ is determined by $\boldsymbol{\gamma}, \boldsymbol{\Gamma}$ and $\boldsymbol{\kappa}, \mathbf{K}$, respectively. However, a possible elimination of $\mu$ and $\mathrm{M}$ is not convenient, especially not in the context of a 3-D analysis.

3. Active and passive transformations. From the pure geometrical point of view, the gradient of surface deformation $\mathbf{f}$ realizes the lifting between the tangent spaces attached to the same material point on the two differential manifolds $\mathscr{M}$ and $m$, respectively. If $\mathbf{n}\left(\theta^{\sigma}, t\right)$ represents a second-order tensor field on the manifold $m$ (more precisely, on a tangent space), which describes any physical property, then as a result of the lifting by the two-point (in the sense of space and time) tensor $\mathbf{f}$ we obtain four new tensor fields attached to

$$
\begin{aligned}
\text { contravariant: } & \mathbf{N}^{* *}\left(\Theta^{\Phi}, T\right)=j \mathbf{f}^{-1} \mathbf{n f}{ }^{-\mathrm{T}}, \\
\text { covariant: } & \mathbf{N}_{* *}\left(\boldsymbol{\Theta}^{\Phi}, T\right)=j \mathbf{f}^{\mathrm{T}} \mathbf{n f}, \\
\text { mixed: } & \left\{\begin{array}{l}
{ }^{*} \mathbf{N}_{*}\left(\Theta^{\Phi}, T\right)=j \mathbf{f}^{-1} \mathbf{n f}, \\
{ }_{*} \mathbf{N}^{*}\left(\Theta^{\Phi}, T\right)=j \mathbf{f}^{\mathrm{T}} \mathbf{n f}{ }^{-\mathrm{T}},
\end{array} \quad \mathbf{n}\left(\theta^{\sigma}, t\right)=\left\{\begin{array}{l}
j^{-1} \mathbf{f N}^{* *} \mathbf{f}^{\mathrm{T}}, \\
j^{-1} \mathbf{f}^{-\mathrm{T}} \mathbf{N}_{* *} \mathbf{f}^{-1}, \\
j^{-1} \mathbf{f}^{*} \mathbf{N}_{*} \mathbf{f}^{-1}, \\
j^{-1} \mathbf{f}^{-\mathrm{T}} \mathbf{N}^{*} \mathbf{f}^{\mathrm{T}} .
\end{array}\right.\right.
\end{aligned}
$$

Here the determinant $j$ plays the role of a coefficient of balance for the field density. Examples of the above operations are the covariant lifts between Eulerian and Lagrangian deformation measures, which can be deduced from (2.3), (2.4), and (2.5),

$$
\left\{\begin{array}{c}
\Gamma \\
\mathbf{K} \\
\mathbf{M}
\end{array}\right\}=\mathbf{f}^{\mathrm{T}}\left\{\begin{array}{l}
\gamma \\
\boldsymbol{\kappa} \\
\mu
\end{array}\right\} \mathbf{f} \text { and }\left\{\begin{array}{l}
\gamma \\
\boldsymbol{\kappa} \\
\mu
\end{array}\right\}=\mathbf{f}^{-\mathrm{T}}\left\{\begin{array}{c}
\Gamma \\
\mathbf{K} \\
\mathbf{M}
\end{array}\right\} \mathbf{f}^{-1} \text {, }
$$


together with the condition of balanced field density, $j=1$. The operation (3.1) preserves symmetry properties and invariant requirements. The lifts of tensor fields by $\mathbf{f}$ carry over the trace of the tensor product of two tensors, $\operatorname{tr}(\mathbf{n} \gamma)$ for instance, only if one tensor is contravariantly and the second covariantly lifted. This last property is fundamental for the determination of appropriate stress measures (see Macveau [16]). Comparing (3.2) and the definitions (2.11) and (2.13) we obtain the contravariant lift rule for stress measures

$$
\left\{\begin{array}{c}
\mathbf{N} \\
\mathbf{M} \\
\mathbf{L}
\end{array}\right\}=j \mathbf{f}^{-1}\left\{\begin{array}{c}
\mathbf{n} \\
\mathbf{m} \\
\mathbf{l}
\end{array}\right\} \mathbf{f}^{-\mathrm{T}} \text { and }\left\{\begin{array}{c}
\mathbf{n} \\
\mathbf{m} \\
\mathbf{l}
\end{array}\right\}=j^{-1} \mathbf{f}\left\{\begin{array}{c}
\mathbf{N} \\
\mathbf{M} \\
\mathbf{L}
\end{array}\right\} \mathbf{f}^{\mathrm{T}},
$$

whereas $j=\operatorname{det} \mathbf{f}$ is used for the balance of stress field density. Within the framework of 3-D continuum, relations such as (3.2) are known as Cosserat mapping [3] and (3.3) as symmetric Piola-Kirchhoff mapping [31, §26]. The formulae are independent of the question: which components of tensors are actually visualized or used for the calculation?

We also consider tensor fields lifted via another two-point tensor, which is connected with the deformation gradient: the orthogonal rotation tensor $\mathbf{r}$ according to (2.10). The rules for the corotational lift are identical with (3.1) when $\mathbf{f}$ is replaced by $\mathbf{r}$. Since $\mathbf{r}^{-1}=\mathbf{r}^{\mathrm{T}}$, it follows from (3.1) that the difference between the covariant, contravariant, and mixed lifts vanishes and the lift becomes a rigid mapping. Therefore we shall call it the rigid lift of tensor fields. Additionally, if $\mathbf{f}=\mathbf{f}^{\mathrm{T}}$ then the rigid lift becomes the identical operation. Also, the rigid lift preserves all algebraic operations such as multiplication, trace, invariants, symmetry, etc.

Both operations of lifting of tensor fields that were discussed above belong to the class of active transformations. It means that as a result of the transformation we obtain a really new physical object described by a new tensor field. The second class belongs to the passive transformations connected with the same physical state but measured by a transformed observer. A known example of a special observer transformation is the "mapping by a shifter." The shifter represents the base multiplication: $g_{\cdot \Sigma}^{\sigma}=\mathbf{a}^{\sigma} \cdot \mathbf{A}_{\Sigma}, g_{\sigma}{ }^{\Sigma}=\mathbf{a}_{\sigma} \cdot \mathbf{A}^{\Sigma}$, which allows us to describe the same object but referred to different observers:

$$
\mathbf{n}=n^{\sigma \omega} \mathbf{a}_{\sigma} \otimes \mathbf{a}_{\omega}=n^{\sigma \omega} g_{\sigma} \cdot{ }^{\Sigma} g_{\omega} \cdot{ }^{\Omega} \mathbf{A}_{\Sigma} \otimes \mathbf{A}_{\Omega} .
$$

Such a kind of passive transformation has been discussed by Naghdi and Wainwright [19], where the active and passive transformations are used with an equal status. Similar effects also can be introduced by applying the kinematically preferred coordinate transformations [30].

We want to point out that the notion of "lifting of tensor fields" that was introduced above includes as a particular case the mappings called pull-back/push-forward operations, performed via the "induced gradient" f only. Since the formulae (3.1) are also applicable to such two-point tensors as those of Eqs. (8.3) and (8.17), the notion "lift of tensors" seems to be more adequate.

4. Material derivative. Conceptually contrary to the previously discussed notions of passive and active transformations, there exists the notion of an exchange of coordinate systems. 
Numerically, the same object described by a tensor or vector can be represented as a different function of a Lagrangian $\left(\Theta^{\Sigma}, T\right)$ or an Eulerian $\left(\theta^{\sigma}, t\right)$ coordinate system. An important example is the displacement vector, represented in two possible ways, $\chi\left(\theta^{\sigma}, t\right)=\mathbf{X}\left(\Theta^{\Sigma}, T\right)$ (Fig. 1). Its temporal change gives a proper measure of velocity. The velocity is defined as the time rate of change of the displacement for a given particle

$$
\mathbf{V}\left(\Theta^{\Sigma}, T\right)=\frac{\partial}{\partial T} X_{\left.\right|_{\Theta=\text { const }}},
$$

whereas $\partial_{t} \chi_{\left.\right|_{\theta=\text { const }}}$ cannot be recognized as a velocity measured by the Eulerian observer. The velocity $\mathbf{v}\left(\theta^{\sigma}, t\right)=\mathbf{V}\left(\Theta^{\Sigma}, T\right)$ in terms of Eulerian coordinates can be found first by replacing $\left(\theta^{\sigma}, t\right)$ in $\chi$ by $\left(\Theta^{\Sigma}, T\right)$ and performing the material derivative (4.1) and then by reexchanging the coordinates [33, §72]. In this paper, for 2-D problems, we shall adopt the concept of a separation of the velocity $\mathbf{v}\left(\theta^{\sigma}, t\right)$ into two parts: the first one is the relative velocity $\mathbf{q}\left(\theta^{\sigma}, t\right)=q^{\varphi} \mathbf{a}_{\varphi}, q^{\varphi}=\partial \theta^{\varphi} /\left.\partial T\right|_{\boldsymbol{\Theta}=\text { const }}$ of the particle with respect to an arbitrary reference system $\left(\theta^{\sigma}, t\right)$, and the second one is a velocity of transport $\mathbf{p}\left(\theta^{\sigma}, t\right)=p^{\varphi} \mathbf{a}_{\varphi}+p^{3} \mathbf{a}_{3}$ of the $\left(\theta^{\sigma}, t\right)$ frame itself. The above split, introduced by Naghdi and Wainwright [19] is extremely significant for moving surfaces where the relative velocity is represented only by a surface vector. Therefore the material derivative of an arbitrary tensor $\boldsymbol{\Phi}\left(\Theta^{\Sigma}, T\right)=\phi\left(\theta^{\sigma}, t\right)$ is calculated by using the relative velocity

$$
\dot{\boldsymbol{\Phi}}=\frac{\partial \Phi}{\partial T} \equiv\left(\frac{\partial \phi}{\partial t}\right)_{\left.\right|_{\theta=\text { const }}} \frac{\partial t}{\partial T}+\frac{\partial \phi}{\partial \theta^{\sigma}}\left(\frac{\partial \theta^{\sigma}}{\partial T}\right)_{\left.\right|_{\theta=\text { const }}} .
$$

The right-hand side of (4.2) is known as the substantial derivative and in hydrodynamics is frequently denoted by the symbol

$$
D \phi / D t=\partial_{t} \phi+q^{\sigma} \partial_{\sigma} \phi,
$$

where $\partial t / \partial T=1$. Since $q^{\sigma} \partial_{\sigma} \phi=\gamma \operatorname{rad} \phi \mathbf{q}=\mathbf{q} \gamma \operatorname{rad}^{\mathrm{T}} \phi$, the relation (4.3) may be rewritten in index free form. Also, we accept here the simplified, often used notation, $\dot{\phi}$, for the material derivative instead of $D / D t$. Thus, taking into account the above settlements, we express (4.3) in the final form

$$
\dot{\phi}\left(\theta^{\sigma}, t\right)=\partial_{t} \phi+\mathbf{q} \gamma \operatorname{rad}^{\mathrm{T}} \phi=\partial_{t} \phi+\mathbf{q} \cdot \nabla_{2} \otimes \phi .
$$

To determine the material derivative of surface tensors and of the two-point deformation gradient tensor $\mathbf{f}$, we have to distinguish between derivatives of components and of bases. Making use of previous definitions we can proceed as follows.

For the derivatives of the components, we obtain

$$
\begin{gathered}
{\dot{\overline{\theta^{\sigma}}}}_{, \Sigma}=\partial_{\Sigma}\left(\frac{\partial \theta^{\sigma}}{\partial T}\right)_{\mid \Theta}=\partial_{\Sigma} q^{\sigma}=\theta_{, \Sigma}^{\varphi} \partial_{\varphi} q^{\sigma}, \\
\dot{\dot{\Theta}^{\Sigma}},{ }_{, \sigma}=-\Theta_{, \rho}^{\Sigma} \Theta_{, \omega}^{\Phi}\left(\dot{\theta^{\rho}}\right)=-\Theta_{, \rho}^{\Sigma} q_{, \sigma}^{\rho} .
\end{gathered}
$$


The local changes of the coordinate base are expressed as

$$
\begin{aligned}
& \partial_{t} \mathbf{a}_{\varphi}=\partial_{t}\left(\partial_{\varphi} \boldsymbol{\theta}\right)=\partial_{\varphi} \mathbf{p}\left(\theta^{\omega}, t\right)=\left(\left.p^{\sigma}\right|_{\varphi}-b_{\varphi}^{\sigma} p^{3}\right) \mathbf{a}_{\sigma}+\left(p_{, \varphi}^{3}+p^{\sigma} b_{\sigma \varphi}\right) \mathbf{a}_{3}, \\
& \partial_{t} \mathbf{a}^{\varphi}=-\left(\left.p^{\varphi}\right|_{\sigma}-b_{\sigma}^{\varphi} p^{3}\right) \mathbf{a}^{\sigma}+\left(a^{\varphi \sigma} p_{, \sigma}^{3}+p^{\sigma} b_{\sigma}^{\varphi}\right) \mathbf{a}_{3}, \\
& \partial_{t} \mathbf{a}_{3}=-\left(p_{, \varphi}^{3}+b_{\sigma \varphi} p^{\sigma}\right) \mathbf{a}^{\varphi}
\end{aligned}
$$

where in the last formula the condition of orthogonality $\mathbf{a}_{3} \cdot \mathbf{a}_{\sigma}=0$ has been used and the covariant derivative $\left.p^{\sigma}\right|_{\varphi}=p_{, \varphi}^{\sigma}-\gamma_{\varphi \omega}^{\sigma} p^{\omega}$ follows from (2.2). According to (4.4) and (4.6), the material derivative of the coordinate base is given by

$$
\begin{aligned}
& \dot{\mathbf{a}}_{\varphi}=\partial_{t} \mathbf{a}_{\varphi}+q^{\sigma} \partial_{\sigma} \mathbf{a}_{\varphi}=\left(\left.p^{\omega}\right|_{\varphi}-b_{\varphi}^{\omega} p^{3}+q^{\sigma} \gamma_{\sigma \varphi}^{\omega}\right) \mathbf{a}_{\omega}+\left(p^{\sigma} b_{\sigma \varphi}+p_{, \varphi}^{3}+q^{\sigma} b_{\sigma \varphi}\right) \mathbf{a}_{3} \\
& \equiv\left(\begin{array}{c}
\sigma \\
\varphi
\end{array}\right) \mathbf{a}_{\sigma}+\left(\begin{array}{c}
3 \\
\varphi
\end{array}\right) \mathbf{a}_{3}, \\
& \dot{\mathbf{a}}^{\varphi} \equiv-\left(\begin{array}{c}
\varphi \\
\sigma
\end{array}\right) \mathbf{a}^{\sigma}-\left(\begin{array}{c}
\varphi \\
3
\end{array}\right) \mathbf{a}^{3}=-\left(\begin{array}{c}
\varphi \\
\sigma
\end{array}\right) \mathbf{a}^{\sigma}+a^{\varphi \sigma}\left(\begin{array}{l}
3 \\
\sigma
\end{array}\right) \mathbf{a}_{3}, \quad \dot{\mathbf{a}}_{3} \equiv-\left(\begin{array}{c}
3 \\
\varphi
\end{array}\right) \mathbf{a}^{\varphi} .
\end{aligned}
$$

These formulae are similar to the Gauss-Weingarten ones (2.2). Therefore, the coefficients $\left(\begin{array}{l}\varphi \\ \sigma\end{array}\right),\left(\begin{array}{l}3 \\ \varphi\end{array}\right)$ may be called the time symbols. The corresponding 3-D symbols were introduced by Naghdi and Wainwright [19].

With (4.5)-(4.7), we obtain further

$$
\begin{gathered}
\dot{a}_{\sigma \omega}=\dot{\mathbf{a}}_{\sigma} \cdot \mathbf{a}_{\omega}+\mathbf{a}_{\sigma} \cdot \dot{\mathbf{a}}_{\omega}=\left(\begin{array}{c}
\rho \\
\sigma
\end{array}\right) a_{\rho \omega}+\left(\begin{array}{c}
\rho \\
\omega
\end{array}\right) a_{\rho \sigma}, \\
\dot{\bar{j}}=\frac{1}{\sqrt{A}} \frac{\dot{[}}{\left[\sqrt{a}\left|\theta_{, \Sigma}^{\sigma}\right|\right]}=-\sqrt{a / A}\left(\left|\theta_{, \Sigma}^{\sigma}\right| \frac{1}{a} \dot{a}+2\left|\theta_{, \Sigma}^{\sigma}\right| \partial_{\omega} q^{\omega}\right)=\left.j v^{\sigma}\right|_{\sigma}=j \delta \mathrm{iv} \mathbf{v} .
\end{gathered}
$$

To establish the material time derivative of a surface tensor, the procedure of material time derivative should be applied simultaneously to components and dyadic base

$$
\begin{aligned}
\dot{\mathbf{n}}=\overline{n^{\sigma \omega} \mathbf{a}_{\sigma} \otimes \mathbf{a}_{\omega}}= & \left(\partial_{t} n^{\sigma \omega}+q^{\varphi} n_{, \varphi}^{\sigma \omega}\right) \mathbf{a}_{\sigma} \otimes \mathbf{a}_{\omega} \\
& +n^{\sigma \omega}\left[\left(\partial_{t} \mathbf{a}_{\sigma}+q^{\varphi} \mathbf{a}_{\sigma, \varphi}\right) \otimes \mathbf{a}_{\omega}+\mathbf{a}_{\sigma} \otimes\left(\partial_{t} \mathbf{a}_{\omega}+q^{\varphi} \mathbf{a}_{\omega, \varphi}\right)\right] \\
\equiv & (D / D t) n^{i j} \mathbf{a}_{i} \otimes \mathbf{a}_{j} \\
= & \left(\delta_{\sigma}^{i} \delta_{\omega}^{j} \dot{n}^{\sigma \omega}+n^{\varphi \omega}\left(\begin{array}{c}
i \\
\varphi
\end{array}\right) \delta_{\omega}^{j}+n^{\sigma \varphi}\left(\begin{array}{c}
j \\
\varphi
\end{array}\right) \delta_{\sigma}^{i}\right) \mathbf{a}_{i} \otimes \mathbf{a}_{j}, \quad i, j=\varphi, \sigma, 3 .
\end{aligned}
$$

It may be observed immediately that the material time derivative destroys the tensor character of $\mathbf{n}$ and that $\dot{\mathbf{n}}$ loses its surface property because it possesses components in the normal direction too. This is one of the main objections against a simple adoption of the three-dimensional rules.

Having distinguished between the material derivative of components and base, the material derivative of the deformation gradient $\mathbf{f}(2.5)$ is expressed as

$$
\begin{aligned}
& \dot{\mathbf{f}}=\overline{\theta_{, \Sigma}^{\sigma} \mathbf{a}_{\sigma} \otimes \mathbf{A}^{\Sigma}}=\left(\partial_{\Sigma} q^{\varphi} \delta_{\varphi}^{i}+\theta_{, \Sigma}^{\varphi}\left(\begin{array}{c}
i \\
\varphi
\end{array}\right)\right) \mathbf{a}_{i} \otimes \mathbf{A}^{\Sigma}=\theta_{, \Sigma}^{\varphi}\left(q_{, \varphi}^{\sigma} \delta_{\sigma}^{i}+\left(\begin{array}{c}
i \\
\varphi
\end{array}\right)\right) \mathbf{a}_{i} \otimes \mathbf{A}^{\Sigma} \\
& =\left[\left(q_{, \varphi}^{\sigma} \delta_{\sigma}^{i}+\left(\begin{array}{c}
i \\
\varphi
\end{array}\right)\right) \mathbf{a}_{i} \otimes \mathbf{a}^{\varphi}\right]\left(\theta_{, \Omega}^{\omega} \mathbf{a}_{\omega} \otimes \mathbf{A}^{\Omega}\right) \equiv \lambda \mathbf{f} .
\end{aligned}
$$


The purely Eulerian tensor $\lambda\left(\theta^{\sigma}, t\right)$ can also be recognized as the surface gradient of velocity

$$
\lambda=\left[\left(q^{\sigma}+p^{\sigma}\right)_{\mid \varphi}-b_{\varphi}^{\sigma} p^{3}\right] \mathbf{a}_{\sigma} \otimes \mathbf{a}^{\varphi}+\left[\left(q^{\omega}+p^{\omega}\right) b_{\omega \varphi}+p_{, \varphi}^{3}\right] \mathbf{a}_{3} \otimes \mathbf{a}^{\varphi}=(\mathbf{q}+\mathbf{p})_{, \varphi} \otimes \mathbf{a}^{\varphi} .
$$

Although $\lambda=\gamma \mathrm{rad} \mathbf{v}$ is in agreement with the 3-D formula, we have $\dot{\mathbf{a}}_{\varphi} \neq \lambda \mathbf{a}_{\varphi}$, but $\dot{\mathbf{a}}_{3}=-\mathbf{a}_{3} \lambda$.

Similarly to (4.9), after using (4.5) and (4.6), the material derivative of the inverse of the deformation gradient takes the form

$$
\dot{\mathbf{f}^{-1}}=-\mathbf{f}^{-1}\left[\mathbf{a} \lambda-\lambda^{\mathrm{T}}(\mathbf{1}-\mathbf{a})\right]
$$

With (4.6) and (4.10) the material derivative of the surface identity tensor can be expressed as

$$
\dot{\mathbf{a}}=(\mathbf{1}-\mathbf{a}) \lambda+\lambda^{\mathrm{T}}(\mathbf{1}-\mathbf{a})
$$

(in contrary to $\mathbf{i}=0$ ). Next, taking into account that the Hamilton operator may also be referred to the Langrangian observer $\gamma \operatorname{rad}(\cdot)=\Gamma \operatorname{rad}(\cdot) \mathbf{f}^{-1}$, the material derivative of the tensor of curvature takes the form

$$
\dot{\mathbf{b}}=-\overline{\gamma \operatorname{rad} \mathbf{a}_{3}}=-\overline{\Gamma \operatorname{rad}\left(\mathbf{a}_{3}\right) \mathbf{f}^{-1}}=\gamma \operatorname{rad}\left(\mathbf{a}_{3} \lambda\right)-\mathbf{b}\left[\lambda-\lambda^{\mathrm{T}}(\mathbf{1}-\mathbf{a})\right]
$$

and, simultaneously,

$$
\begin{aligned}
& \dot{\gamma}=\frac{1}{2}\left[\dot{\mathbf{a}}+\left(\lambda^{\mathrm{T}} \mathbf{a}-(\mathbf{1}-\mathbf{a}) \boldsymbol{\lambda}\right) \mathbf{f}^{-\mathrm{T}} \mathbf{A} \mathbf{f}^{-1}+\mathbf{f}^{-\mathrm{T}} \mathbf{A f}^{-1}\left(\mathbf{a} \lambda-\lambda^{\mathrm{T}}(\mathbf{1}-\mathbf{a})\right)\right] \\
& \dot{\boldsymbol{\kappa}}=-\left[\dot{\mathbf{b}}+\left(\boldsymbol{\lambda}^{\mathrm{T}} \mathbf{a}-(\mathbf{1}-\mathbf{a}) \lambda\right) \mathbf{f}^{-\mathrm{T}} \mathbf{B} \mathbf{f}^{-1}+\mathbf{f}^{-\mathrm{T}} \mathbf{B} \mathbf{f}^{-1}\left(\mathbf{a} \lambda-\lambda^{\mathrm{T}}(\mathbf{1}-\mathbf{a})\right)\right] \\
& \dot{\boldsymbol{\mu}}=\frac{1}{2}\left[\dot{\mathbf{b}}^{2}+\left[\lambda^{\mathrm{T}} \mathbf{a}-(\mathbf{1}-\mathbf{a}) \lambda\right) \mathbf{f}^{-\mathrm{T}} \mathbf{B}^{2} \mathbf{f}^{-1}+\mathbf{f}^{-\mathrm{T}} \mathbf{B}^{2} \mathbf{f}^{-1}\left(\mathbf{a} \lambda-\lambda^{\mathrm{T}}(\mathbf{1}-\mathbf{a})\right)\right] .
\end{aligned}
$$

5. Convective rates of surface tensors. As we mentioned previously,we restrict ourselves to the consideration of objective rates induced by the convective lifts of tensor fields. Therefore we remain in the domain of the validity of Oldroyd's concept and our numerous results presented in this section have their three-dimensional analogue. Nevertheless, since a surface possesses its own specification, our aim is the definition of surface rates starting from a more fundamental concept.

Since the pioneering paper of Oldroyd [22], it is known that the four possibilities of lift of tensor field (3.1) induce also four possible convective derivatives. According to tradition, we shall reserve the superposed pyramid $(\stackrel{\Delta}{\circ})$ for covariant convective derivative and superposed "inverse pyramid" $(\stackrel{\nabla}{)}$ ) for contravariant one. We recall that the names of "contra-" and "covariant" have nothing to do with components of tensors, which means that the formulae look identical in any holonomic base.

Putting, for the moment, the coefficient of balance of field density equal to one, 
$j=1$, from (3.1), (4.9), and (4.11), we immediately obtain

$$
\begin{aligned}
& \left.\stackrel{\nabla}{\mathbf{n}}=\overline{\mathbf{f}\left(\mathbf{f}^{-1} \mathbf{n f}\right.} \mathbf{f}^{-\mathbf{T}}\right) \mathbf{f}^{\mathrm{T}} \\
& =\mathbf{f}\left[-\mathbf{f}^{-1}\left(\mathbf{a} \lambda-\lambda^{\mathrm{T}}(\mathbf{1}-\mathbf{a})\right) \mathbf{n}+\mathbf{f}^{-1} \dot{\mathbf{n}} \mathbf{f}^{-\mathrm{T}}-\mathbf{f}^{-1} \mathbf{n}\left(\lambda^{\mathrm{T}} \mathbf{a}+(\mathbf{1}-\mathbf{a}) \boldsymbol{\lambda}\right)\right] \mathbf{f}^{\mathrm{T}} \\
& \equiv \mathbf{a n a}-\mathbf{a} \lambda \mathbf{n}-\mathbf{n} \lambda^{\mathrm{T}} \mathbf{a} \text {, }
\end{aligned}
$$

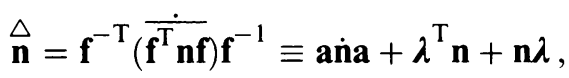

$$
\begin{aligned}
& \mathfrak{n}^{\prime}=\mathbf{f}\left(\overline{\mathbf{f}^{-1} \mathbf{n f}}\right) \mathbf{f}^{-1} \equiv \mathbf{a n a}-\mathbf{a} \boldsymbol{\lambda} \mathbf{n}+\mathbf{n} \boldsymbol{\lambda}, \\
& \grave{n}=\mathbf{f}^{-\mathrm{T}}\left(\overline{\mathbf{f}^{\mathrm{T}} \mathbf{n} \mathbf{f}^{-\mathrm{T}}}\right) \mathbf{f}^{\mathrm{T}} \equiv \mathbf{a n a}+\lambda^{\mathrm{T}} \mathbf{n}-\mathbf{n} \lambda^{\mathrm{T}} \mathbf{a},
\end{aligned}
$$

the contra-, covariant, and two mixed convective derivatives. A particular form of the contravariant rate $(5.1)_{1}$, when $j=\operatorname{det} \mathbf{f}$, is obtained in the form of the Truesdell derivative

$$
\underset{\mathbf{n}}{\mathrm{T}}=j^{-1} \mathbf{f}\left(\overline{j \mathbf{f}^{-1} \mathbf{n f} \mathbf{f}^{-\mathrm{T}}}\right) \mathbf{f}^{\mathrm{T}}=\mathbf{a n a}-\mathbf{a} \boldsymbol{\lambda} \mathbf{n}-\mathbf{n} \lambda^{\mathrm{T}} \mathbf{a}+\mathbf{n} \delta \mathrm{iv} \mathbf{v} .
$$

The 3-D analogue of (5.2) was introduced by Truesdell [31, $\S 55$ bis] owing to his examination of Cauchy's stress flux principle. Notice that all convective derivatives (5.1) remain surface tensors. It means that the presence of the surface identity tensor a in (5.1) follows from primary principles.

It seems to be reasonable to examine the rates of surface strain measures $\gamma, \boldsymbol{\kappa}$, $\boldsymbol{\mu}$. Starting from $(5.1)_{2}, j=1$, and (4.15) the covariant rate of the Almansi-Hamel tensor $\gamma$ is expressed by

$$
\stackrel{\Delta}{\gamma}=\mathbf{a} \dot{\gamma} \mathbf{a}+\lambda^{\mathrm{T}} \gamma+\gamma \lambda=\frac{1}{2}\left(\lambda^{\mathrm{T}} \mathbf{a}+\mathbf{a} \lambda\right) \equiv \boldsymbol{\delta}_{0}=\delta_{0 \sigma \omega} \mathbf{a}^{\sigma} \otimes \mathbf{a}^{\omega}=\mathbf{f}^{-\mathrm{T}} \dot{\boldsymbol{\Gamma}} \mathbf{f}^{-1}
$$

and, similarly,

$$
\begin{aligned}
& \stackrel{\Delta}{\boldsymbol{\kappa}}=\mathbf{a} \dot{\boldsymbol{\kappa}} \mathbf{a}+\lambda^{\mathrm{T}} \boldsymbol{\kappa}+\boldsymbol{\kappa} \lambda=-\mathbf{a} \gamma \operatorname{rad}\left(\mathbf{a}_{3} \lambda\right)-\lambda^{\mathrm{T}} \mathbf{b} \equiv \boldsymbol{\delta}_{1}=\delta_{1 \sigma \omega} \mathbf{a}^{\sigma} \otimes \mathbf{a}^{\omega}=\mathbf{f}^{-\mathrm{T}} \dot{\mathbf{K}} \mathbf{f}^{-1}, \\
& \stackrel{\Delta}{\boldsymbol{\mu}}=\mathbf{a} \boldsymbol{\mu} \mathbf{a}+\lambda^{\mathrm{T}} \boldsymbol{\mu}+\boldsymbol{\mu} \lambda=\frac{1}{2}\left[\gamma \operatorname{rad}^{\mathrm{T}}\left(\mathbf{a}_{3} \lambda\right) \mathbf{b}+\mathbf{b} \gamma \operatorname{rad}\left(\mathbf{a}_{3} \lambda\right)\right] \equiv \boldsymbol{\delta}_{2}=\delta_{2 \sigma \omega} \mathbf{a}^{\sigma} \otimes \mathbf{a}^{\omega} .
\end{aligned}
$$

Considering (5.3) and (5.4) we can conclude that for surfaces $\stackrel{\Delta}{\gamma}=\boldsymbol{\delta}_{0}, \overleftrightarrow{\kappa}=\boldsymbol{\delta}_{1}$, and $\stackrel{\Delta}{\mu}=\delta_{2}$ correspond to the rate of deformation tensor $\mathbf{d}=\operatorname{sym}(\operatorname{grad} \mathbf{v})$ in threedimensional continuum [31, §21]. The 3-D analogue of $\hat{\gamma}=\mathbf{f}^{-\mathrm{T}} \dot{\boldsymbol{\Gamma}} \mathbf{f}^{-1}$ has been examined by Cosserat [3] as well as by Murnaghan [18]. The greatest advantage of these covariant rates is their linear dependence on the velocity gradient.

The objective covariant acceleration can be calculated by repeating the procedure

$$
\begin{aligned}
& \stackrel{\Delta}{\gamma}^{\Delta}=\stackrel{\boldsymbol{\delta}}{0}_{0}=\mathbf{f}^{-\mathrm{T}} \dot{\boldsymbol{\Gamma}} \mathbf{f}^{-1}=\frac{1}{2}\left[\dot{\lambda}^{\mathrm{T}} \mathbf{a}+\mathbf{a} \dot{\boldsymbol{\lambda}}+\lambda^{\mathrm{T}} \lambda^{\mathrm{T}} \mathbf{a}+\mathbf{a} \lambda \boldsymbol{\lambda}+2 \lambda^{\mathrm{T}} \mathbf{a} \lambda\right] \\
& =\frac{1}{2}\left[\dot{\lambda}_{\sigma \varphi}+\dot{\lambda}_{\varphi \sigma}+\lambda_{. \varphi}^{\omega} \lambda_{\omega \sigma}+\lambda_{\varphi \omega} \lambda_{. \sigma}^{\omega}+2 \lambda_{. \varphi}^{\omega} \lambda_{\omega \sigma}\right] \mathbf{a}^{\varphi} \otimes \mathbf{a}^{\sigma}, \\
& \Delta^{\Delta}=\stackrel{\Delta}{\boldsymbol{\delta}}_{1}=\mathbf{f}^{-\mathrm{T}} \dot{\mathbf{K}} \mathbf{f}^{-1}=\mathbf{a} \dot{\boldsymbol{\delta}}_{1} \mathbf{a}+\lambda^{\mathrm{T}} \boldsymbol{\delta}_{1}+\boldsymbol{\delta}_{1} \boldsymbol{\lambda}, \\
& \stackrel{\Delta}{\boldsymbol{\mu}}^{\Delta}=\stackrel{\Delta}{\boldsymbol{\delta}}_{2}=\mathbf{f}^{-\mathrm{T}} \dot{\mathbf{M f}}^{-1}=\mathbf{a} \dot{\boldsymbol{\delta}}_{2} \mathbf{a}+\lambda^{\mathrm{T}} \boldsymbol{\delta}_{2}+\boldsymbol{\delta}_{2} \lambda .
\end{aligned}
$$


These formulae are, of course, more complex nonlinear functions of the velocity gradient. Furthermore, making use of $(5.1)_{1},(4.15)$, and (4.11), the contravariant rates of $\gamma, \boldsymbol{\kappa}, \boldsymbol{\mu}$ can be calculated as follows $(j=1)$ :

$$
\begin{aligned}
& \stackrel{\nabla}{\gamma}=\mathbf{a} \dot{\gamma} \mathbf{a}-\mathbf{a} \lambda \boldsymbol{\gamma}-\gamma \lambda^{\mathrm{T}} \mathbf{a}=\boldsymbol{\delta}_{0}-2 \boldsymbol{\delta}_{0} \gamma-2 \gamma \boldsymbol{\delta}_{0}, \\
& \stackrel{\nabla}{\boldsymbol{\kappa}}=\mathbf{a} \dot{\boldsymbol{\kappa}} \mathbf{a}-\mathbf{a} \boldsymbol{\alpha} \boldsymbol{\kappa}-\boldsymbol{\kappa} \lambda^{\mathrm{T}} \mathbf{a} \equiv \boldsymbol{\delta}_{1}-2 \boldsymbol{\delta}_{0} \boldsymbol{\kappa}-2 \boldsymbol{\kappa} \boldsymbol{\delta}_{0}, \\
& \stackrel{\nabla}{\boldsymbol{\mu}}=\mathbf{a} \boldsymbol{\mu} \mathbf{a}-\mathbf{a} \boldsymbol{\lambda} \boldsymbol{\mu}-\boldsymbol{\mu} \lambda^{\mathrm{T}} \mathbf{a} \equiv-\nabla^{2}-2 \boldsymbol{\delta}_{0} \boldsymbol{\mu}-2 \boldsymbol{\mu} \boldsymbol{\delta}_{0} .
\end{aligned}
$$

If we are looking for another objective rate that is conserving the symmetry property but simultaneously eliminating the difference between contra- and covariant rates, then we take the symmetric part of $(5.1)_{1}$ and $(5.2)_{2}$ and obtain $(j=1)$

$$
\begin{aligned}
\stackrel{\square}{\mathbf{n}}=\frac{1}{2}(\stackrel{\overrightarrow{\mathbf{n}}+\stackrel{\nabla}{\mathbf{n}})}{ } & =\frac{1}{2}(\grave{\mathbf{n}}+\mathbf{n})=\mathbf{a} \mathbf{n} \mathbf{a}+\frac{1}{2}\left(\boldsymbol{\lambda}^{\mathrm{T}} \mathbf{a}-\mathbf{a} \boldsymbol{\lambda}\right) \mathbf{n}+\frac{1}{2} \mathbf{n}\left(\mathbf{a} \boldsymbol{\lambda}-\lambda^{\mathrm{T}} \mathbf{a}\right) \\
& \equiv \mathbf{a n a} \mathbf{a}-\boldsymbol{\omega}_{0} \mathbf{n}+\mathbf{n} \boldsymbol{\omega}_{0},
\end{aligned}
$$

the definition of a surface derivative of Zaremba-Jaumann type. In (5.7) the surface vorticity tensor

$$
\boldsymbol{\omega}_{0}=-\boldsymbol{\omega}_{0}^{\mathrm{T}}=\omega_{0} \boldsymbol{\varepsilon}=\frac{1}{2}\left(\mathbf{a} \lambda-\lambda^{\mathrm{T}} \mathbf{a}\right), \quad \boldsymbol{\varepsilon} \equiv(-\mathbf{1} \times \mathbf{1}) \mathbf{a}_{3}, \quad \boldsymbol{\varepsilon}^{2}=-\mathbf{a},
$$

was introduced in a natural way. With respect to the property of the skew-symmetry of $\boldsymbol{\varepsilon}^{\mathrm{T}}=-\boldsymbol{\varepsilon}$, this tensor has only one independent component $\omega_{0}$.

A few previous works, among them Guo [8] and Truesdell and Tupin [33, §148], introduced the Zaremba-Jaumann derivative using other primary arguments. Especially Guo [8] very highly appreciated the role of the Zaremba-Jaumann derivative and made the original effort for a generalization of this notion. In light of his result the Zaremba-Jaumann derivative belongs to the class of "constitutive derivatives." Only now do we know about the serious faults of it, for example, the oscillation of stresses in the plane shear problem.

The surface derivatives of Zaremba-Jaumann type of the Almansi-Hamel strain measures are

$$
\begin{aligned}
& \stackrel{\square}{\gamma}=\mathbf{a} \dot{\gamma} \mathbf{a}-\boldsymbol{\omega}_{0} \gamma+\gamma \boldsymbol{\omega}_{0}=\boldsymbol{\delta}_{0}-\boldsymbol{\delta}_{0} \gamma-\gamma \boldsymbol{\delta}_{0}, \\
& \stackrel{\square}{\boldsymbol{\kappa}}=\mathbf{a} \dot{\boldsymbol{\kappa}} \mathbf{a}-\omega_{0} \boldsymbol{\kappa}+\boldsymbol{\kappa} \omega_{0}=\boldsymbol{\delta}_{1}-\boldsymbol{\delta}_{0} \boldsymbol{\kappa}-\boldsymbol{\kappa} \boldsymbol{\delta}_{0}, \\
& \stackrel{\square}{\mu}=\mathbf{a} \boldsymbol{\mu} \mathbf{a}-\omega_{0} \boldsymbol{\mu}+\boldsymbol{\mu} \omega_{0}=\delta_{2}-\delta_{0} \boldsymbol{\mu}-\boldsymbol{\mu} \boldsymbol{\delta}_{0}, \quad \stackrel{\mathrm{a}}{\mathbf{a}}=0 .
\end{aligned}
$$

It may be observed that without causing ambiguity all these convective rates can be collected into one family of convective derivatives

$$
\frac{D^{m}}{D t} \mathbf{n}=\mathbf{a n a}-\boldsymbol{\omega}_{0} \mathbf{n}+\mathbf{n} \boldsymbol{\omega}_{0}+m\left(\boldsymbol{\delta}_{0} \mathbf{n}+\mathbf{n} \boldsymbol{\delta}_{0}\right)
$$

where for $m=-1, m=1, m=0$ the covariant, contravariant, Zaremba-Jaumann derivatives are particular cases.

Another convective derivative is induced by the rigid lift of tensors using in (5.1) the rotation tensor $\mathbf{r}$. In this case no difference between contravariant, covariant, and mixed derivatives can be observed:

$$
\overrightarrow{\mathbf{n}}=\mathbf{r}^{-\mathrm{T}} \frac{\dot{\cdot}}{\left(\mathbf{r}^{\mathrm{T}} \mathbf{n r}\right) \mathbf{r}^{-1}} \equiv \overline{\mathbf{r}\left(\mathbf{r}^{-1} \mathbf{n r}\right) \mathbf{r}^{\mathrm{T}}}=\hat{\mathbf{n}} \equiv \overrightarrow{\mathbf{n}} .
$$


If by $\boldsymbol{\Omega}_{0}=\mathbf{a}\left(\dot{\mathbf{r}}{ }^{-1}\right) \mathbf{a}=\boldsymbol{\Omega}_{0} \boldsymbol{\varepsilon}, \boldsymbol{\Omega}_{0}=-\boldsymbol{\Omega}_{0}^{\mathrm{T}}$ we denote the surface material spin tensor, then "the rigid Zaremba-Jaumann" derivative (5.11) is evaluated as

$$
\ddot{\mathbf{n}}=\mathbf{a} \mathbf{n} \mathbf{a}-\boldsymbol{\Omega}_{0} \mathbf{n}+\mathbf{n} \boldsymbol{\Omega}_{0} .
$$

Since, according to the polar decomposition (2.10), the following relation holds:

$$
\boldsymbol{\omega}_{0}=\boldsymbol{\Omega}_{0}+\frac{1}{2} \mathbf{a r}\left(\dot{\cup} \cup^{-1}-\cup^{-1} \dot{U}\right) \mathbf{r a},
$$

the material spin $\boldsymbol{\Omega}_{0}$ differs, in general, from $\boldsymbol{\omega}_{0}$, and therefore

$$
\bar{\gamma}=\stackrel{q}{\gamma}+\left(\boldsymbol{\omega}_{0}-\boldsymbol{\Omega}_{0}\right) \boldsymbol{\gamma}+\boldsymbol{\gamma}\left(\boldsymbol{\Omega}_{0}-\boldsymbol{\omega}_{0}\right), \quad \overline{\mathbf{a}}=0 .
$$

Yet another possibility follows if the stretch $U$ is used in the process of lifting of tensor fields (3.1). To determine such a derivative we first observe that instead of $U$ rather a combination of the two-point tensors $\mathbf{f}$ and $\mathbf{r}$ should be applied. From $(3.1)_{1,2}$ two kinds of Biot-like tensors are defined,

$$
\begin{aligned}
& \mathbf{N}^{\mathrm{B}}=\frac{1}{2}\left({ }^{*} \mathbf{N}_{*} U^{-1}+U^{-1}{ }_{*} \mathbf{N}^{*}\right)=\frac{1}{2} j\left(\mathbf{f}^{-1} \mathbf{n r}+\mathbf{r}^{\mathrm{T}} \mathbf{n r}{ }^{-\mathrm{T}}\right), \\
& \mathbf{N}_{\mathrm{B}}=\frac{1}{2}\left({ }_{*} \mathbf{N}^{*} U^{-1}+U^{-1}{ }^{*} \mathbf{N}_{*}\right)=\frac{1}{2} j\left(\mathbf{f}^{\mathrm{T}} \mathbf{n r}+\mathbf{r}^{\mathrm{T}} \mathbf{n f}\right) .
\end{aligned}
$$

Next a contra- and a covariant derivative,

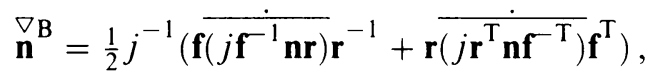

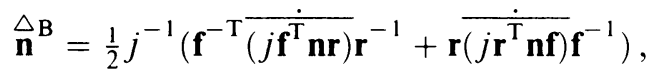

are defined by using the suitable definitions of $\boldsymbol{\omega}_{0}, \boldsymbol{\Omega}_{0}, \boldsymbol{\delta}_{0}$, hence leading to

$$
\begin{aligned}
& \nabla_{\mathbf{n}} \mathrm{B}=\mathbf{a n a}+\mathbf{n} \delta \mathbf{i v} \mathbf{v}+\left(-\boldsymbol{\Omega}_{0}+\mathbf{a} \lambda\right) \mathbf{n}+\mathbf{n}\left(\boldsymbol{\Omega}_{0}-\lambda^{\mathrm{T}} \mathbf{a}\right), \\
& \triangle_{\mathbf{B}} \mathrm{B}=\mathbf{a n a} \mathbf{a}+\mathbf{n} \delta \mathbf{i v} \mathbf{v}+\left(-\boldsymbol{\Omega}_{0}+\lambda^{\mathrm{T}} \mathbf{a}\right) \mathbf{n}+\mathbf{n}\left(\boldsymbol{\Omega}_{0}+\mathbf{a} \lambda\right) .
\end{aligned}
$$

Since $\mathbf{a} \lambda=\boldsymbol{\delta}_{0}+\boldsymbol{\omega}_{0}, \lambda^{\mathrm{T}} \mathbf{a}=\boldsymbol{\delta}_{0}-\boldsymbol{\omega}_{0}$, these last relations can be written similarly to (5.10) as a family of "B-derivatives"

$$
\frac{D^{\mathrm{B}}}{D t} \mathbf{n}=\mathbf{a n a}+\mathbf{n} \delta \operatorname{iv} \mathbf{v}-\left(\boldsymbol{\Omega}_{0}+\boldsymbol{\omega}_{0}\right) \mathbf{n}+\mathbf{n}\left(\boldsymbol{\Omega}_{0}+\boldsymbol{\omega}_{0}\right)+\mathrm{B}\left(\boldsymbol{\delta}_{0} \mathbf{n}+\mathbf{n} \boldsymbol{\delta}_{0}\right) .
$$

For $\mathrm{B}=-1$ and $\mathrm{B}=1$ we have the contra- and covariant derivatives, respectively. However, $B=0$ leads to a Zaremba-Jaumann type of derivative and such case was introduced by Green and McInnis [7] in the 3-D context.

6. Objective rates in the constitutive equations. Before considering constitutive relations for surfaces, we want to recall some early results of continuum mechanics. 
Natanson [20] was probably the first who gave a rigorous three-dimensional extension of the Maxwell model of viscoelasticity. In the period 1892-1903 the main goal of Natanson's efforts was to give a rigorous mathematical foundation of irreversible thermodynamics. In particular, he tried to describe the old Helmholtz concept of a unification of thermodynamics, electrodynamics, and continuum mechanics. In 1896 he proposed a "thermokinetic variational principle" as the foundation of the Helmholtz unified field theory. Therefore, starting to work on the Maxwell model, Natanson looked for other applications of his principle. Keeping in mind the requirements of irreversible thermodynamics, he made serious efforts toward a split of mechanical properties of deformable continuum into reversible and irreversible parts. In particular, he considered "the relaxed state" connected with some kind of "time irreversible derivative." Not solving this ambitious program, he investigated finally a linearized, superposable model of a spring and a dashpot.

The nonlinear improvement of the three-dimensional Maxwell model has been given by Zaremba [37], who replaced the material time derivative of stress tensors by a derivative of the type (5.7). Natanson appreciated this solution positively as the first step towards a finite viscoelasticity. However, from the point of view of irreversible thermodynamics, Zaremba's solution was unsatisfactory-in Zaremba's objective derivative both irreversible and reversible agendae take part equally. Natanson did not accept the "corotational" derivative and he, up to the end, preferred his thermodynamic point of view.

Irreversible thermodynamics was also the departure point for a paper of Eckart [6]. He introduced a decomposition of the rate of strain tensor into anelastic and elastic parts associated with a local relaxation. Unfortunately, the thermodynamical line of Natanson-Eckart has not been generally accepted in the field of Rational Thermodynamics. In particular, Truesdell [31, §22] has commented that the name of "anelasticity tensor" is an "unhappy term" and interpreted it as "a reference rate tensor." Nevertheless, numerous theories based on different concepts of a relaxed, intermediate state are still subjects of actual research. Recently, within the framework of finite elastoplasticity, the irreversible objective time derivative has been proposed by Stumpf [29].

In turn, Zaremba's solution was developed and applied by Jaumann [12] first and later revindicated and fully justified within the framework of Rational Mechanics in Noll's dissertation [21]. This "corotational" derivative, but in Jaumann's version, has been proposed for plasticity also by Prager [24].

Independent of a controversial discussion about the Zaremba-Jaumann derivative, a quite different approach to constructing the Maxwell model was considered by Truesdell $[31, \S \S 56,81 ; 32]$. Overcoming the original Natanson line towards a finite viscoelasticity, Truesdell as a starting point uses an assumption about a linear superposition of elastic and viscotic agendae ("superposition theories"). In Truesdell's proposition an elastic element (spring) should be described by a hypoelastic constitutive equation satisfying simultaneously Cauchy's flux principle and the objectivity requirements. Taking into account Zaremba's solution [36, Eq. (32)], he proposed a satisfactory form of the hypoelastic constitutive equation [31, p. 605, Eq. (56.2)] 


$$
\stackrel{\mathrm{T}}{\mathbf{n}}=\lambda_{E} \operatorname{tr}(\stackrel{\triangle}{\gamma}) \mathbf{a}+2 \mu_{E} \stackrel{\triangle}{\gamma},
$$

where, in our 2-dimensional version, $\mathbf{n}$ is the derivative (5.2) and $\lambda_{E}, \mu_{E}$ are moduli of elasticity. Note that this formula connects the contravariant rate of Cauchy type stress tensor and the covariant rate of Almansi-Hamel strain tensor.

According to our remark that the trace of the product of two tensors remains unchanged under simultaneous lift of both tensors, for example $\operatorname{tr}(\mathrm{T} \Delta \vec{\gamma})=\operatorname{tr}(\dot{\mathbf{N}} \dot{\Gamma})$, Truesdell's proposition is additionally justified. Adopting this approach for the 3-D continuum, we propose constitutive equations for the viscoelastic flexible surface in the form

$$
\begin{aligned}
& \mathbf{n}+t_{n}^{-1} \underset{\mathbf{n}}{\mathrm{T}}=\mathrm{A}_{00}\left(\stackrel{\Delta}{\gamma}+\varepsilon_{n} \stackrel{\Delta}{\gamma}^{\Delta}\right)+\mathrm{A}_{01}\left(\stackrel{\Delta}{\boldsymbol{\kappa}}+\varepsilon_{n m} \stackrel{\Delta}{\boldsymbol{\kappa}}\right), \\
& \mathbf{m}+t_{m}^{-1} \stackrel{\mathbf{T}}{\mathbf{m}}=\mathrm{A}_{10}\left(\stackrel{\Delta}{\boldsymbol{\gamma}}+\varepsilon_{n m} \stackrel{\Delta}{\gamma}\right)+\mathrm{A}_{11}\left(\stackrel{\Delta}{\boldsymbol{\kappa}}+\varepsilon_{m} \stackrel{\Delta}{\boldsymbol{\kappa}}\right) .
\end{aligned}
$$

This is a generalized Maxwell-Voigt form of linear constitutive relations for the range of moderate rates of strains. The fourth-order tensor coefficients $\mathrm{A}_{i j}, i, j=0,1$, may be supposed to be functions of the stress and moment tensors $\mathbf{n}$ and $\mathbf{m}$, respectively. When the flexible and extensional properties of the surface are decomposed, the cross coefficients $A_{01}, A_{10}$ vanish. In analogy to the 3-D case, the coefficients $t_{n}, t_{m}$ may be called the stress relaxation time and $\varepsilon_{n}, \varepsilon_{n m}, \varepsilon_{m}$ strain retardation time constants [22, 31].

From Eq. (6.2) a family of relations can be obtained governing the material behaviour of viscoelastic flexible surfaces. When $\mathbf{m}, \mathbf{n}$ and the strain accelerations $\Delta \Delta, \Delta{ }^{\Delta}$ are zero simultaneously, (6.2) takes the form of a hypoelastic material. When $\underset{\mathbf{m}}{\mathrm{T}}, \mathbf{n}$ and $\gamma, \boldsymbol{\kappa}$ are zero simultaneously, the linear equations for Newtonian fluid are obtained. In turn, when $\hat{\gamma}^{\Delta}, \Delta^{\Delta}$ vanish, (6.2) represents the linear Maxwell fluid equations; finally, when $t_{n}, t_{m} \rightarrow \infty$, the viscoelastic Kelvin-Voigt fluid model is obtained.

Whereas it is generally accepted in 3-D continuum mechanics to use covariant derivatives for the rate of deformation tensors, different convective time derivatives are used in the constitutive relations for the stress rates. Oldroyd [22, §4] and Noll

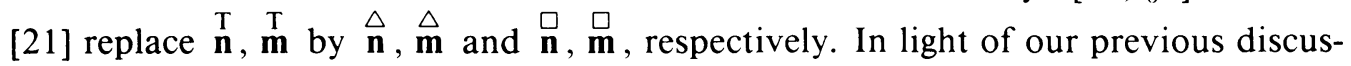
sion, we do not agree with both formulations.

7. Special cases. In Sec. 4 we introduced the general concept of a decomposition of the absolute velocity $\mathbf{v}$ into a relative velocity $\mathbf{q}$ with respect to the reference frame $\left(\theta^{\sigma}, t\right)$ and the velocity of transport $\mathbf{p}$ of the frame itself.

Now we want to consider two special cases.

7.1. Hencky convective coordinates. When the moving system moves in common with the surface, $\left\{\theta^{\sigma}, t\right\}$ becomes the convected system, $\theta^{\sigma}=\delta_{\Sigma}^{\sigma} \Theta^{\Sigma}, t=T$ and [8]

$$
\mathbf{q}=0, \quad \mathbf{p}=\mathbf{v} .
$$

The induced base vectors $\mathbf{a}_{\sigma}=\delta_{\sigma}^{\Sigma}\left(\mathbf{A}_{\Sigma}+\mathbf{X}_{, \Sigma}\right) \equiv \mathbf{a}_{\Sigma} \delta_{\sigma}^{\Sigma}$, denoted shortly by $\mathbf{a}_{\Sigma}$, contain the information about deformation and motion. This simplifies our formulae 
significantly, in particular (2.5), (2.11), (4.10),

$$
\begin{aligned}
\mathbf{a} & =\mathbf{a}_{\Sigma} \otimes \mathbf{a}^{\Sigma}=a_{\Sigma \Omega} \mathbf{a}^{\Sigma} \otimes \mathbf{a}^{\Omega}, \quad \mathbf{b}=\left(-\mathbf{a}_{3}\right)_{, \Sigma} \otimes \mathbf{a}^{\Sigma}=b_{\Sigma \Omega} \mathbf{a}^{\Sigma} \otimes \mathbf{a}^{\Omega}, \quad a_{\Sigma \Omega}=\mathbf{a}_{\Sigma} \cdot \mathbf{a}_{\Omega}, \\
\mathbf{f} & =\mathbf{a}_{\Sigma} \otimes \mathbf{A}^{\Sigma}, \quad \quad \quad \gamma=\frac{1}{2}\left(a_{\Sigma \Omega}-A_{\Sigma \Omega}\right) \mathbf{a}^{\Sigma} \otimes \mathbf{a}^{\Omega}=\gamma_{\Sigma \Omega^{2}} \mathbf{a}^{\Sigma} \otimes \mathbf{a}^{\Omega}, \\
\lambda & =\gamma \operatorname{rad} \mathbf{p}=\left(p_{\| \Sigma}^{\Omega}-b_{\Sigma}^{\Omega} p^{3}\right) \mathbf{a}_{\Omega} \otimes \mathbf{a}^{\Sigma}+\left(p^{\Omega} b_{\Omega \Sigma}+p_{, \Sigma}^{3}\right) \mathbf{a}_{3} \otimes \mathbf{a}^{\Sigma} \\
& =\lambda_{\cdot \Sigma}^{\Omega} \mathbf{a}_{\Omega} \otimes \mathbf{a}^{\Sigma}+\lambda_{\cdot \Sigma}^{3} \mathbf{a}_{3} \otimes \mathbf{a}^{\Sigma} .
\end{aligned}
$$

The material time derivatives $(4.7)$ reduce to

$$
\dot{\mathbf{a}}_{\Sigma}=\lambda \mathbf{a}_{\Sigma}, \quad \dot{\mathbf{a}}^{\Sigma}=-\lambda \mathbf{a}^{\Sigma}, \quad \dot{\mathbf{a}}_{3}=-\mathbf{a}_{3} \lambda .
$$

Formula (4.4) leads to $\dot{\phi} \equiv \partial_{t} \phi$ and (4.8) takes the form

$$
\dot{\mathbf{n}}=\left(\dot{n}^{\Sigma \Omega}+n^{\Phi \Omega} \lambda_{\cdot \Phi}^{\Sigma}+n^{\Sigma \Phi} \lambda_{\cdot \Phi}^{\Omega}\right) \mathbf{a}_{\Sigma} \otimes \mathbf{a}_{\Omega}+n^{\Sigma \Omega} \lambda_{\cdot \cdot^{3}}^{3} \mathbf{a}_{3} \otimes \mathbf{a}_{\Sigma}+\mathbf{n}^{\Sigma \Omega} \lambda_{\cdot \Omega^{3}}^{3} \mathbf{a}_{\Sigma} \otimes \mathbf{a}_{3} .
$$

Taking into account the form of $\lambda$ according to (7.2) and

$$
\begin{aligned}
& \stackrel{\Delta}{\gamma}=\boldsymbol{\delta}_{0}=\frac{1}{2}\left(p_{\Omega \| \Sigma}+p_{\Sigma \| \Omega}-2 b_{\Omega \Sigma} p^{3}\right) \mathbf{a}^{\Omega} \otimes \mathbf{a}^{\Sigma}=\delta_{0 \Omega \Sigma} \mathbf{a}^{\Omega} \otimes \mathbf{a}^{\Sigma}, \\
& \stackrel{\widehat{\kappa}}{=}=\boldsymbol{\delta}_{1}=-\left(\lambda_{3 \Omega \| \Sigma}+\lambda_{\Phi \Sigma} b_{\Omega}^{\Phi}\right) \mathbf{a}^{\Omega} \otimes \mathbf{a}^{\Sigma}=\delta_{1 \Omega \Sigma} \mathbf{a}^{\Omega} \otimes \mathbf{a}^{\Sigma},
\end{aligned}
$$

we obtain, with (7.4) and (5.7), simplified formulae for the Zaremba-Jaumann derivative

$$
\stackrel{\square}{\mathbf{n}}=\left(\dot{n}^{\Sigma \Omega}+n^{\Phi \Omega} \delta_{0 \cdot \Phi}^{\Sigma}+n^{\Sigma \Phi} \delta_{0 \cdot \Phi}^{\Omega}\right) \mathbf{a}_{\Sigma} \otimes \mathbf{a}_{\Omega}
$$

and for the Truesdell derivative

$$
\underset{\mathbf{n}}{\mathrm{T}}=\left(\dot{n}^{\Sigma \Omega}+n^{\ddot{\Sigma} \Omega} \delta_{0 \cdot \Phi}^{\Phi}\right) \mathbf{a}_{\Sigma} \otimes \mathbf{a}_{\Omega}, \quad \dot{n}^{\Sigma \Omega}=\partial_{t} n^{\Sigma \Omega} .
$$

For the 3-D continuum, Lehmann [15] pointed out the surprising form of (7.6), depending on the symmetric part $\boldsymbol{\delta}_{0}$ and not on the antisymmetric part $\boldsymbol{\omega}_{0}$. Note that the relative simple form of Eqs. (7.6) and (7.7) is compensated by the presence of [26]

$$
\gamma_{\Sigma \Phi}^{\Omega}=\Gamma_{\Sigma \Phi}^{\Omega}+a^{\Omega \Delta}\left(\gamma_{\Delta \Sigma \mid \Phi}+\gamma_{\Phi \Delta \mid \Sigma}-\gamma_{\Sigma \Phi \mid \Delta}\right)
$$

the "deformed" Christoffel symbols in the computation of the velocity gradient $v_{\| \Omega}^{\Sigma}=$ $v_{, \Omega}^{\Sigma}-v^{\Phi} \gamma_{\Phi \Omega}^{\Sigma}$.

7.2. Motionless reference space. When the reference space is motionless and the system $\left\{\theta^{\sigma}, t\right\}$ is fixed, the velocity consists of two parts, namely,

$$
\mathbf{p}\left(\theta^{\sigma}, t\right)=p^{3} \mathbf{a}_{3}, \quad \mathbf{q}\left(\theta^{\sigma}, t\right)=q^{\sigma} \mathbf{a}_{\sigma} .
$$


Thus, (7.9), (4.6), and (4.7) lead to

$$
\begin{gathered}
\partial_{t} \mathbf{a}_{\varphi}=-b_{\varphi}^{\sigma} p^{3} \mathbf{a}_{\sigma}+p_{, \varphi}^{3} \mathbf{a}_{3}, \quad \partial_{t} \mathbf{a}_{3}=-p_{, \varphi}^{3} \mathbf{a}^{\varphi}, \\
\partial_{t} \mathbf{a}^{\varphi}=b_{\sigma}^{\varphi} p^{3} \mathbf{a}^{\sigma}+a^{\varphi \sigma} p_{,{ }^{3}} \mathbf{a}_{3}, \\
\left(\begin{array}{c}
\sigma \\
\varphi
\end{array}\right)=-b_{\varphi}^{\sigma} p^{3}+q^{\omega} \gamma_{\omega \varphi}^{\sigma}, \quad\left(\begin{array}{c}
3 \\
\varphi
\end{array}\right)=p_{, \varphi}^{3}+q^{\omega} b_{\omega \varphi}, \\
\partial_{t} a_{\sigma \omega}=-2 p^{3} b_{\sigma \omega}, \quad \partial_{t} a^{\sigma \omega}=2 p^{3} b^{\sigma \omega}, \quad \dot{\mathbf{a}}=\left(\begin{array}{c}
3 \\
\varphi
\end{array}\right)\left(\mathbf{a}_{3} \otimes \mathbf{a}^{\varphi}+\mathbf{a}^{\varphi} \otimes \mathbf{a}_{3}\right), \\
\dot{a}_{\sigma \omega}=-2 p^{3} b_{\sigma \omega}+q^{\rho}\left(\gamma_{\rho \sigma}^{\varphi} a_{\varphi \omega}+\gamma_{\rho \omega}^{\varphi} a_{\varphi \sigma}\right) .
\end{gathered}
$$

Furthermore with Eq. (7.10), the material time derivative (4.8) takes the form

$$
\begin{aligned}
\dot{\mathbf{n}}= & \left(\partial_{t} n^{\sigma \omega}+q^{\varphi} n_{\mid \varphi}^{\sigma \omega}\right) \mathbf{a}_{\sigma} \otimes \mathbf{a}_{\omega}+q^{\varphi} n^{\sigma \omega}\left(b_{\varphi \sigma} \mathbf{a}_{3} \otimes \mathbf{a}_{\omega}+b_{\varphi \omega} \mathbf{a}_{\sigma} \otimes \mathbf{a}_{3}\right) \\
& +n^{\sigma \omega}\left(p_{,{ }_{\sigma}}^{3} \mathbf{a}_{3} \otimes \mathbf{a}_{\omega}+p_{, \omega}^{3} \mathbf{a}_{\sigma} \otimes \mathbf{a}_{3}\right)-n^{\sigma \omega} p^{3}\left(b_{\sigma}^{\varphi} \mathbf{a}_{\varphi} \otimes \mathbf{a}_{\omega}+b_{\omega}^{\varphi} \mathbf{a}_{\sigma} \otimes \mathbf{a}_{\varphi}\right) \\
= & \partial_{t} \mathbf{n}+\mathbf{q} \gamma \mathrm{rad}^{\mathrm{T}} \mathbf{n},
\end{aligned}
$$

and in virtue of (7.10), and (7.11), the Zaremba-Jaumann derivative is obtained as

$$
\stackrel{\mathrm{n}}{\mathbf{n}}=\left(\partial_{t} n^{\sigma \omega}+q^{\varphi} n_{\mid \varphi}^{\sigma \omega}-n^{\varphi \omega} p^{3} b_{\varphi}^{\sigma}+n^{\sigma \varphi} p^{3} b_{\varphi}^{\omega}+\omega_{0} \varepsilon_{. .}^{\sigma \varphi} n_{\rho}^{\omega} .+\omega_{0} \varepsilon_{. .}^{\varphi \omega} n_{. \rho}^{\sigma}\right) \mathbf{a}_{\sigma} \otimes \mathbf{a}_{\omega} .
$$

The formulae (7.10) and (7.11) are identical with those presented by Povstenko and Podstigach [23].

8. The boundary curve. There is a characteristic feature of low-dimensional mechanics and physics that 4-D and 3-D quantities are functions of one or two coordinates only. Before going to the boundary, let us recall the example of a 3-D stress tensor as function of surface coordinates

$$
\mathcal{N}\left(\theta^{\varphi}, t\right)=n^{\sigma \omega} \mathbf{a}_{\sigma} \otimes \mathbf{a}_{\omega}+n^{3 \omega}\left(\mathbf{a}_{3} \otimes \mathbf{a}_{\omega}+\mathbf{a}_{\omega} \otimes \mathbf{a}_{3}\right)+n^{33} \mathbf{a}_{3} \otimes \mathbf{a}_{3} .
$$

A well-known simple example of $\mathscr{N}$ is the pressure tensor within a capillary layer $\mathscr{N}=p_{\|} \mathbf{a}+p_{\perp} \mathbf{a}_{3} \otimes \mathbf{a}_{3}, \mathbf{a}=a_{\sigma \omega} \mathbf{a}^{\sigma} \otimes \mathbf{a}^{\omega}$. A quantity like $\mathscr{N}$, usually describing the phenomena from an "outside world," can be a function of the surface coordinates with the gradient

$$
\begin{aligned}
\gamma \operatorname{rad} \mathscr{N} & =\mathscr{N}{ }_{\omega} \otimes \mathbf{a}^{\omega} \\
& =\mathscr{N} \cdot{ }_{\omega}^{\varphi \sigma} \mathbf{a}_{\varphi} \otimes \mathbf{a}_{\sigma} \otimes \mathbf{a}^{\omega}+\mathscr{N} \cdot{ }_{\omega}^{3 \sigma}\left(\mathbf{a}_{3} \otimes \mathbf{a}_{\sigma}-\mathbf{a}_{\sigma} \otimes \mathbf{a}_{3}\right) \otimes \mathbf{a}^{\omega}+\mathscr{N} \cdot{ }_{\omega}^{33} \mathbf{a}_{3} \otimes \mathbf{a}_{3} \otimes \mathbf{a}^{\omega},
\end{aligned}
$$

where

$$
\begin{aligned}
\mathscr{N}_{\cdot{ }_{\omega}}^{\varphi \sigma} & =n_{\mid \omega}^{\varphi \sigma}-n^{3 \varphi} b_{\omega}^{\sigma}-n^{3 \sigma} b_{\omega}^{\varphi}, \\
\mathscr{N}^{3 \sigma}{ }_{\omega}^{3 \sigma} & =n_{\mid \omega}^{3 \sigma}+n^{\sigma \nu} b_{\nu \omega}-n^{33} b_{\omega}^{\sigma}, \\
\mathscr{N}^{33}{ }_{\omega}^{33} & =n_{, \omega}^{33}+2 n^{3 \nu} b_{\nu \omega},
\end{aligned}
$$

and a function of time with the material time derivatives (4.8) and (4.7). Nevertheless, it is an open question: how do we construct a three-dimensional objective derivative by using only well-defined surface quantities like $\lambda, \delta_{0}, \delta_{1}, \delta_{2}, \omega_{0}$ ? Since the 
discrepancy of dimensions cannot be removed, there exists some kind of arbitrariness in passing from two to three dimensions. It can be seen that a simple model follows from an extension of the gradient of deformation $\mathbf{f}$ to a three-dimensional one,

$$
\mathbf{F}^{*}=\mathbf{f}+\mathbf{a}_{3} \otimes \mathbf{A}^{3} \delta_{3}^{3}=\mathbf{f}+\mathbf{a}_{3} \otimes \mathbf{A}^{3},
$$

which does not involve any new parameters; it still contains $\mathbf{f}$ and its algebraic combinations. By virtue of $\dot{\mathbf{a}}_{3}=-\mathbf{a}_{3} \lambda$ and $\dot{\mathbf{F}}^{*}=\dot{\mathbf{f}}+\dot{\mathbf{a}}_{3} \otimes \mathbf{A}^{3}$, the 3-D tensor $\boldsymbol{\Lambda}$, analog of $\lambda$, is defined to be

$$
\mathbf{\Lambda}=\dot{\mathbf{F}}^{*}\left(\mathbf{F}^{*}\right)^{-1}=\lambda-\lambda^{\mathrm{T}}(\mathbf{1}-\mathbf{a})=\lambda_{\omega \sigma} \mathbf{a}^{\omega} \otimes \mathbf{a}^{\sigma}+\lambda_{3 \omega}\left(\mathbf{a}^{3} \otimes \mathbf{a}^{\omega}-\mathbf{a}^{\omega} \otimes \mathbf{a}^{3}\right) .
$$

To determine objective rates a relation must be assumed between the tensor $\mathbf{F}^{*}$ and a two-point tensor proper for a lift of $\mathcal{N}$ analogous to (3.1) and (5.1). For instance, using our proposition of $\mathbf{F}^{*}$ and (8.4), we obtain with $(5.1)_{1,2}$ the objective contraand covariant rates $\left(\mathbf{F}^{*} \mathbf{F}^{*-1}=\mathbf{F}^{*-1} \mathbf{F}^{*}=\mathbf{1}\right)$

$$
\stackrel{\nabla}{N}^{3}=\dot{\mathscr{N}}-\boldsymbol{\Lambda} \mathscr{N}-\mathscr{N} \boldsymbol{\Lambda}^{\mathrm{T}}, \quad \stackrel{\Delta}{N}^{3}=\dot{\mathscr{N}}+\boldsymbol{\Lambda}^{\mathrm{T}} \mathscr{N}+\mathscr{N} \boldsymbol{\Lambda} .
$$

Here the derivatives are entirely expressed by the 2-D gradient of velocity only. However, if for any reason we prefer a richer model of surface continuum, for instance,

$$
\mathbf{F}^{*}=\mathbf{f}+\boldsymbol{\theta}_{1} \otimes \mathbf{A}^{3}, \quad \boldsymbol{\theta}_{1}=\theta_{1}^{\sigma} \mathbf{a}_{\sigma}+\theta_{1}^{3} \mathbf{a}_{3},
$$

then $\boldsymbol{\Lambda}=\boldsymbol{\Lambda}\left(\gamma \mathrm{rad} \mathbf{v}, \dot{\boldsymbol{\theta}}_{1}\right)$ contains additional quantities like $\boldsymbol{\Theta}$ and, what is unusual, the objective time derivatives (8.5) are determined by using also other parameters than the velocity gradient.

Now keeping in mind the properties of the above-mentioned problem, let us go to the boundary. Here, similar to the above-discussed stress tensor (8.1), the difference between dimension of the surface stress tensor

$$
\mathbf{n}(s, t)=n_{s \jmath} \mathbf{a}_{s} \otimes \mathbf{a}_{s}+n_{s \nu}\left(\mathbf{a}_{s} \otimes \boldsymbol{\nu}+\boldsymbol{\nu} \otimes \mathbf{a}_{s}\right)+n_{\nu \nu} \boldsymbol{\nu} \otimes \boldsymbol{\nu}
$$

and the dimension of the base manifold, i.e., the boundary curve $c=\partial m$, is equal to one. Therefore the analogous level of arbitrariness may be observed. Let us first recall the geometrical relations associated with the boundary. Let $c=\partial m$ be any regular closed boundary curve parameterized by its arc length s (Fig. 1). Then $\left\{\mathbf{a}_{s}, \boldsymbol{\nu}, \mathbf{a}_{3}\right\}$ is its Darboux orthogonal triad

$$
\mathbf{a}_{s}=(d / d s) \boldsymbol{\theta}(s, t)=\boldsymbol{\theta}, s, \quad \boldsymbol{\nu}=\mathbf{a}_{s} \times \mathbf{a}_{3}, \quad \mathbf{a}_{3}=\boldsymbol{\nu} \times \mathbf{a}_{s}, \quad \mathbf{a}_{s}=\mathbf{a}_{3} \times \boldsymbol{\nu}
$$

and the following Poisson-Darboux differential equations hold [35]:

$$
\begin{aligned}
& \mathbf{a}_{s, s}=\sigma_{s} \mathbf{a}_{3}-\kappa_{s} \boldsymbol{\nu}=\omega_{s} \times \mathbf{a}_{s}, \\
& \mathbf{a}_{3, s}=-\sigma_{s} \mathbf{a}_{s}+\tau_{s} \nu=\omega_{s} \times \mathbf{a}_{3}, \\
& \boldsymbol{\nu}_{, s}=\kappa_{s} \mathbf{a}_{s}-\tau_{s} \mathbf{a}_{3}=\omega_{s} \times \boldsymbol{\nu},
\end{aligned}
$$

where the Darboux vector $\boldsymbol{\omega}_{s}=\sigma_{s} \nu+\tau_{s} \mathbf{a}_{s}+\kappa_{s} \mathbf{a}_{3}$ is a function of the normal curvature $\left(\sigma_{s}\right)$, the geodesic torsion $\left(\tau_{s}\right)$, and the geodesic curvature $\left(\kappa_{s}\right)$. The identical relations hold on the boundary, where $\mathbf{A}_{\mathscr{S}}=\boldsymbol{\Theta}, \mathscr{S}, \frac{d}{d \mathscr{S}}\left\{\mathbf{A}_{\mathscr{S}}, \mathrm{N}, \mathbf{A}_{3}\right\}=$ $\boldsymbol{\omega}_{\mathscr{S}} \times\left\{\mathbf{A}_{\mathscr{S}}, \mathbf{N}, \mathbf{A}_{3}\right\}, \boldsymbol{\omega}_{\mathscr{S}}=\sigma_{\mathscr{S}} \mathbf{N}+\tau_{\mathscr{S}} \mathbf{A}_{\mathscr{S}}+\kappa_{\mathscr{S}} \mathbf{A}_{3}$, etc. (Fig. 1). 
The deformation of $c$ is described by

$$
\mathbf{f}_{c}(s, \mathscr{S}, t, T)=\frac{d}{d \mathscr{S}}(\boldsymbol{\theta}) \otimes \mathbf{A}_{\mathscr{S}}=\frac{d s}{d \mathscr{S}} \boldsymbol{\theta},{ }_{s} \otimes \mathbf{A}_{\mathscr{S}}=s, \mathfrak{S}_{s} \otimes \mathbf{A}_{\mathscr{S}},
$$

a 1-D gradient of the position vector. The single component $s, \mathscr{S}$ plays the role of the stretch of the boundary curve. According to the concept of decomposition of the velocity into relative velocity $\mathbf{q}$ and velocity of transport $\mathbf{p}$, we have

$$
\mathbf{v}(\varsigma, t)=\mathbf{p}+\mathbf{q}=\left(p_{\lrcorner}+q_{\lrcorner}\right) \mathbf{a}_{\lrcorner}+p_{\nu} \boldsymbol{\nu}+p_{3} \mathbf{a}_{3} .
$$

With the Eulerian rule of time differentiation (4.4) we obtain

$$
\begin{aligned}
\left(\frac{\partial}{\partial t} \mathbf{a}_{\lrcorner}\right)_{\mid \mathscr{S}} \equiv \partial_{t} \mathbf{a}_{s}= & \left.\frac{d}{d s} \mathbf{p}(\lrcorner, t\right)=\left(p_{\lrcorner, s}-p_{3} \sigma_{s}+p_{\nu} \kappa_{s}\right) \mathbf{a}_{s} \\
& +\left(p_{\nu, s}-p_{s} \kappa_{s}+p_{3} \tau_{s}\right) \boldsymbol{\nu}+\left(p_{3, s}-p_{\nu} \tau_{s}+p_{s} \sigma_{s}\right) \mathbf{a}_{3} .
\end{aligned}
$$

The local derivative of $\mathbf{a}_{3}$ has only two components $\left(\partial_{t} \mathbf{a}_{3}\right) \cdot \boldsymbol{\nu}$ and $\left(\partial_{t} \mathbf{a}_{3}\right) \cdot \mathbf{a}_{j}$. From $\mathbf{a}_{3} \cdot \mathbf{a}_{s}=0$ it follows that $\left(\partial_{t} \mathbf{a}_{3}\right) \cdot \mathbf{a}_{s}=-\left(\partial_{t} \mathbf{a}_{s}\right) \cdot \mathbf{a}_{3}=-\mathbf{p}, \cdot \mathbf{a}_{3}$; however, $\left(\partial_{t} \mathbf{a}_{3}\right) \cdot$ $\boldsymbol{\nu}=-\left(d \mathbf{p} / d_{s_{\nu}}\right) \cdot \mathbf{a}_{3} \equiv v_{\nu}$ remain undetermined by virtue of the impossibility of differentiation in the direction perpendicular to $\mathbf{a}_{s}$ and $\mathbf{a}_{3}$. Therefore $\partial_{t} \mathbf{a}_{3}$ and $\partial_{t} \boldsymbol{\nu}$ are now also a function of the additional fourth parameter $v_{z}$

$$
\begin{aligned}
& \partial_{t} \boldsymbol{\nu}=-\left(p_{\nu, s}-p_{\jmath} \kappa_{\jmath}+p_{3} \tau_{s}\right) \mathbf{a}_{\jmath}+\left(p_{\lrcorner, s}-p_{3} \sigma_{\lrcorner}+p_{\nu} \kappa_{\jmath}\right) \boldsymbol{\nu}-v_{\nu} \mathbf{a}_{3}, \\
& \partial_{t} \mathbf{a}_{3}=-\left(p_{3, s}-p_{\nu} \boldsymbol{\tau}_{s}+p_{s} \sigma_{s}\right) \mathbf{a}_{s}+v_{\nu} \nu \text {. }
\end{aligned}
$$

According to the 1-D version of (4.4) $\dot{\phi} \equiv \partial_{t} \phi+\mathbf{q} \cdot \nabla_{s} \otimes \phi, \nabla_{s}=\mathbf{a}_{s} d / d s$, and with (8.11) and (8.12) the material derivative of the Darboux triad is

$$
\begin{aligned}
& \dot{\mathbf{a}}_{s}=\partial_{t} \mathbf{a}_{s}+q_{s} \mathbf{a}_{s, s} \\
& =\left(p_{s, s}-p_{3} \sigma_{s}+p_{\nu} \kappa_{s}\right) \mathbf{a}_{s}+\left(p_{\nu, s}-p_{s} \kappa_{s}+p_{3} \tau_{s}-q_{s} \kappa_{s}\right) \boldsymbol{\nu} \\
& +\left(p_{3, s}-p_{\nu} \tau_{s}+p_{s} \sigma_{s}+q_{s} \sigma_{s}\right) \mathbf{a}_{3} \\
& \equiv\left(\begin{array}{l}
\jmath \\
s
\end{array}\right) \mathbf{a}_{s}+\left(\begin{array}{l}
\nu \\
\jmath
\end{array}\right) \nu+\left(\begin{array}{l}
3 \\
s
\end{array}\right) \mathbf{a}_{3} \\
& \dot{\mathbf{a}}_{3}=-\left(p_{3, s}+p_{\nu} \tau_{s}+p_{s} \sigma_{s}+q_{s} \sigma_{s}\right) \mathbf{a}_{s}+\left(v_{\nu}+q_{s} \tau_{s}\right) \boldsymbol{\nu} \equiv\left(\begin{array}{l}
\jmath \\
3
\end{array}\right) \mathbf{a}+\left(\begin{array}{l}
\nu \\
3
\end{array}\right) \nu, \\
& \dot{\boldsymbol{\nu}}=-\left(p_{\nu, s}-p_{s} \kappa_{s}+p_{3} \tau_{s}-q_{s} \kappa_{s}\right) \mathbf{a}_{s}+\left(p_{\jmath, s}-p_{\jmath} \sigma_{s}+p_{\nu} \kappa_{s}\right) \boldsymbol{\nu}-\left(v_{\nu}+q_{s} \tau_{\jmath}\right) \mathbf{a}_{3} \\
& \equiv\left(\begin{array}{l}
\jmath \\
\nu
\end{array}\right) \mathbf{a}_{s}+\left(\begin{array}{l}
\nu \\
\nu
\end{array}\right) \nu+\left(\begin{array}{l}
3 \\
\nu
\end{array}\right) \mathbf{a}_{3} \text {. }
\end{aligned}
$$

From the above relations it follows that the material derivative of any surface tensor 
$\mathbf{n}(s, t)$ of the type (8.7) is represented by the formula

$$
\begin{aligned}
\dot{\mathbf{n}}= & \left(\partial_{t} n_{s s}+q_{s} n_{s s, s}+2 n_{s s}\left(\begin{array}{l}
s \\
s
\end{array}\right)+n_{s \nu}\left(\begin{array}{l}
s \\
\nu
\end{array}\right)+n_{\nu s}\left(\begin{array}{l}
s \\
\nu
\end{array}\right)\right) \mathbf{a}_{s} \otimes \mathbf{a}_{s} \\
& +\left(\partial_{t} n_{s \nu}+q_{s} n_{s \nu, s}+n_{s s}\left(\begin{array}{l}
\nu \\
s
\end{array}\right)+n_{s \nu}\left(\begin{array}{l}
s \\
s
\end{array}\right)+n_{s \nu}\left(\begin{array}{l}
\nu \\
\nu
\end{array}\right)+n_{\nu \nu}\left(\begin{array}{l}
s \\
\nu
\end{array}\right)\right) \mathbf{a}_{s} \otimes \nu \\
& +\left(\partial_{t} n_{\nu s}+q_{s} n_{\nu s, s}+n_{s s}\left(\begin{array}{l}
\nu \\
s
\end{array}\right)+n_{\nu s}\left(\begin{array}{l}
\nu \\
\nu
\end{array}\right)+n_{\nu s}\left(\begin{array}{l}
s \\
s
\end{array}\right)+n_{\nu \nu}\left(\begin{array}{l}
s \\
\nu
\end{array}\right)\right) \nu \otimes \mathbf{a}_{s} \\
& +\left(\partial_{t} n_{\nu \nu}+q_{s} n_{\nu \nu, s}+n_{s \nu}\left(\begin{array}{l}
\nu \\
s
\end{array}\right)+n_{\nu s}\left(\begin{array}{l}
\nu \\
s
\end{array}\right)+2 n_{\nu \nu}\left(\begin{array}{l}
\nu \\
\nu
\end{array}\right)\right) \nu \otimes \nu \\
& +\left(n_{s s}\left(\begin{array}{l}
3 \\
s
\end{array}\right)+n_{\nu s}\left(\begin{array}{l}
3 \\
\nu
\end{array}\right)\right) \mathbf{a}_{3} \otimes \mathbf{a}_{s}+\left(n_{s \nu}\left(\begin{array}{l}
3 \\
s
\end{array}\right)+n_{\nu \nu}\left(\begin{array}{l}
3 \\
\nu
\end{array}\right)\right) \mathbf{a}_{3} \otimes \nu \\
& +\left(n_{s \rho}\left(\begin{array}{l}
3 \\
s
\end{array}\right)+n_{s \nu}\left(\begin{array}{l}
3 \\
\varphi
\end{array}\right)\right) \mathbf{a}_{s} \otimes \mathbf{a}_{3}+\left(n_{\nu s}\left(\begin{array}{l}
3 \\
s
\end{array}\right)+n_{\nu \nu}\left(\begin{array}{l}
3 \\
\nu
\end{array}\right)\right) \nu \otimes \mathbf{a}_{3} .
\end{aligned}
$$

Making use of (8.13) and definition (8.9), the material time derivative of the 1-D deformation gradient $\mathbf{f}_{c}$ can be evaluated

$$
\begin{aligned}
& \dot{\mathbf{f}}_{c}=j_{, \mathscr{S}} \mathbf{a}_{s} \otimes \mathbf{A}_{\mathscr{S}}+{ }_{\jmath}, \dot{\mathbf{a}}_{\jmath} \otimes \mathbf{A}_{\mathscr{S}}=\left[\frac{d}{d \mathscr{S}}\left(\partial_{t}\right) \delta_{\jmath}^{i}+{ }_{\jmath}, \mathscr{S}\left(\begin{array}{l}
i \\
\jmath
\end{array}\right)\right] \mathbf{a}_{i} \otimes \mathbf{A}_{\mathscr{S}} \\
& =\left[\left(\delta_{s}^{i} q_{s, s}+\left(\begin{array}{l}
i \\
s
\end{array}\right)\right) \mathbf{a}_{i} \otimes \mathbf{a}_{s}\right]\left[s_{, \mathscr{S}} \mathbf{a}_{s} \otimes \mathbf{A}_{\mathscr{S}}\right] \equiv \lambda_{c} \mathbf{f}_{c}, \quad i=s, \nu, 3 .
\end{aligned}
$$

By inspection of the last formula it can be seen that $\lambda_{c}$ can also be expressed as a $1-\mathrm{D}$ gradient of velocity

$$
\begin{aligned}
\boldsymbol{\lambda}_{c}= & \mathbf{v} \otimes \nabla_{s}=(\mathbf{p}+\mathbf{q})_{, s} \otimes \mathbf{a}_{s} \\
= & \left(q_{\lrcorner, s}+p_{s, s}-p_{3} \sigma_{s}+p_{\nu} \kappa_{s}\right) \mathbf{a}_{s} \otimes \mathbf{a}_{s} \\
& +\left(p_{\nu, s}-p_{s} \kappa_{s}+p_{3} \tau_{s}-q_{s} \kappa_{s}\right) \boldsymbol{\nu} \otimes \mathbf{a}_{s} \\
& +\left(p_{3, s}-p_{\nu} \tau_{s}+p_{s} \sigma_{s}+q_{s} \sigma_{s}\right) \mathbf{a}_{3} \otimes \mathbf{a}_{s} .
\end{aligned}
$$

Now, repeating the previously used argumentation, we are going to endow the model of boundary line with an additional structure. Similar to (8.3), the simplest extension is

$$
\mathbf{f}^{*}=\mathbf{f}_{c}+\boldsymbol{\nu} \otimes \mathbf{N}
$$

which leads to

$$
\lambda^{*}\left(\mathbf{v}, v_{\nu}\right)=\dot{\mathbf{f}}^{*}\left(\mathbf{f}^{*-1}\right)=\lambda_{c}+\left(\begin{array}{l}
\jmath \\
\nu
\end{array}\right) \mathbf{a}_{s} \otimes \nu+\left(\begin{array}{l}
\nu \\
\nu
\end{array}\right) \boldsymbol{\nu} \otimes \nu+\left(\begin{array}{l}
3 \\
\nu
\end{array}\right) \mathbf{a}_{3} \otimes \nu
$$

a 2-D tensor expressed in terms of four independent quantities.

Finally, for a surface tensor of type (8.7) and $\mathbf{a}=\mathbf{a}_{s} \otimes \mathbf{a}_{s}+\boldsymbol{\nu} \otimes \boldsymbol{\nu}$, after using $(8.14),(8.15)$, and $(5.1)_{1,2}$ we obtain the contra- and covariant objective derivatives

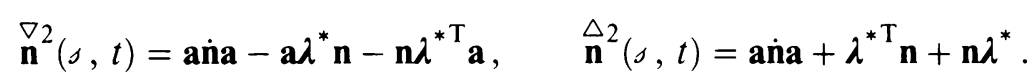

The formulae (8.19) describe the objective derivatives for a generalized model of 1-D continuum. 
It is an open question; how do we find a generalized model of the line whose velocity and $v_{\nu}$ will be described as material derivatives of four parameters? It seems that the best candidate for such a model is the Kirchhoff line [13]. This line is thought of as generalized 1-D continuum with three components of displacement $\chi(\lrcorner, t)$ and one component of the rotation vector $\psi(s, t)=\psi \mathbf{a}_{s}$. It is also possible to express the four velocity parameters $\mathbf{v}(\varsigma, t), v_{\nu}(\varsigma, t)$ as functions of the material derivative of the generalized displacements $\dot{\chi}$ and $\dot{\psi}$. These relations will be discussed elsewhere [28].

However, for many surface phenomena like the propagation of a shock front [14], the material line $c$ can be thought of as nonmaterial moving on a surface $m$. Then the normal component of velocity $p_{\nu}$ plays the role identical with that of $p_{3}$ for the nonmaterial surfaces and may be a base for the formulation of displacement derivatives. It seems also to be easy to adopt the notion of displacement derivative for this case and to work out a theory based on Bowen and Wang's results [2].

Our considerations are far from being complete. Many other possibilities, starting from those based on the elementary concept (Durban and Baruch [5]) and ending with those dealing with the relativistic surface (Zorski [38]) are discussed in a more general context by Bampi and Morro [1].

\section{REFERENCES}

[1] F. Bampi and A. Morro, Objectivity and objective time derivatives in continuum physics, Found. Phys. 10, 905-920 (1980)

[2] R. M. Bowen and C.-C. Wang, On displacement derivatives, Quart. Appl. Math. 29, 29-39 (1971)

[3] E. Cosserat and F. Cosserat, Sur la théorie de l'élasticité, Ann. Toulouse 10, 1-116 (1896)

[4] B. A. Cotter and R. S. Rivlin, Tensors associated with time-dependent stress, Quart. Appl. Math. 13, 177-182 (1955)

[5] D. Durban and M. Baruch, Natural stress rate, Quart. Appl. Math. 35, 55-61 (1977)

[6] C. Eckart, The thermodynamics of irreversible process. IV: The theory of elasticity and anelasticity, Phys. Rev. 73, 373-382 (1948)

[7] A. E. Green and B. C. McInnis, Generalized hypo-elasticity, Proc. Roy. Soc. Edinburgh Sect. A 67, 220-230 (1967)

[8] Guo Zhong-Heng, Time derivatives of tensor fields in non-linear continuum mechanics, Arch. Mech. (Arch. Mech. Stos.) 15, 131-163 (1963)

[9] M. E. Gurtin, Multiphase thermomechanics with interfacial structure. 1. Heat conducting and the capillary balance law, Arch. Rational. Mech. Anal. 104, 195-221 (1988)

[10] M. E. Gurtin and A. I. Murdoch, A continuum theory of elastic material surfaces, Arch. Rational Mech. Anal. 57, 291-323 (1974)

[11] D. Y. Hsieh, Lagrangian formulation of bubble dynamics, Quart. Appl. Math. 33, 115-130 (1975)

[12] G. Jaumann, Geschlossenes System physikalischer und chemischer Differentialgesetze, Sitzungsber. Berlin. Akad. Wiss. Wien (IIa) 120, 385-530 (1911)

[13] G. Kirchhoff, Über das Gleichgewicht und die Bewegung eines unendlich dünnen elastischen Stabes, J. Reine Angew. Math. 56, 285-313 (1859)

[14] W. Kosinski, Field singularities and wave analysis in continuum mechanics, Ellis Horwood Ltd, Chichester, 1986

[15] Th. Lehmann, Formänderungen eines klassischen Kontinuums in vierdimensionaler Darstellung, Proc. 19 Internat. Congress Appl. Mech., H. Görtler, ed., Springer-Verlag, Berlin, 1964, pp. 376382

[16] D. B. Macveau, Die Elementararbeit in einem Kontinuum und die Zuordnung von Spannungs- und Verzerrungstensoren, Z. Angew. Math. Phys. 19, 157-185 (1968)

[17] E. F. Masur, On the definition of stress rate, Quart. Appl. Math. 19, 160-163 (1961)

[18] F. D. Murnaghan, Finite deformations of an elastic solid, Amer. J. Math. 59, 235-260 (1937) 
[19] P. M. Naghdi and W. L. Wainwright, On the time derivative of tensors in mechanics of continua, Quart. Appl. Math. 19, 95-109 (1961)

[20] L. Natanson, On the laws of viscosity, Philos. Mag. 2, 342-356 (1901)

[21] W. Noll, On the continuity of solid and fluid states, J. Rational Mech. Anal. 4, 3-81 (1955)

[22] J. G. Oldroyd, On the formulation of rheological equations of state, Proc. Roy. Soc. London Ser. A 200, 523-541 (1950)

[23] Iu. Z. Povstenko and Ia. S. Podstigach, Time differentiation of tensors defined on a surface moving through a three-dimensional Euclidean space, Prikl. Mat. Mekh. 47, 1038-1044 (1983)

[24] W. Prager, An elementary discussion of definitions of stress rate, Quart. Appl. Math. 18, 403-407 (1961)

[25] L. I. Sedov, Concepts of different rates of change of tensors, Prikl. Mat. Mekh. 14, 393-398 (1960)

[26] H. Stumpf, General concept of the analysis of thin elastic shells, Z. Angew. Math. Mech. 66, 337350 (1986)

[27] H. Stumpf and K. Ch. Le, Variational principles of nonlinear fracture mechanics, Acta Mech. 83, 25-37 (1990)

[28] H. Stumpf, On the balance equations of a surface via descent from 3-D continuum (to appear)

[29] __ On the shakedown analysis in finite elastoplasticity, Internat. J. Plasticity (to appear)

[30] T. Y. Thomas, Kinematically preferred coordinate systems, Proc Nat. Acad. Sci. U. S. A. 41, $762-770$ (1955)

[31] C. Truesdell, The mechanical foundation of elasticity and fluid dynamics, J. Rational Mech. Anal. 1, 125-300 (1952); errata 2, 593-616 (1953)

[32] _ - The simplest rate theory of pure elasticity, Comm. Pure Appl. Math. 8, 123-132 (1955)

[33] C. Truesdell and R. A. Tupin, The classical field theories, S. Flügge, ed., Handbuch der Physik, Vol. III/I, Springer-Verlag, Berlin, 1960, pp. 226-793

[34] L. M. Truskinovskii, Equilibrium phase interfaces, Sov. Phys. Dokl. 27, 551-553 (1982)

[35] C. E. Weatherburn, Differential geometry of three dimensions, Cambridge Univ. Press, Cambridge, vol. 1 (1927), vol. 2 (1928)

[36] S. Zaremba, Sur une généralisation de la théorie classique de la viscosité, Bull. Internat. l'Acad. Sci. Lett. Cracovie (Cl. Sci. Math. Natur.), A 380-403 (1903)

[37] A. Zmitrowicz, Mathematical description of anisotropic friction, Internat. J. Solids Structures 25, 837-862 (1989)

[38] H. Zorski, Rigid motion of relativistic surfaces, Continuum Mechanics and Related Problems of Analysis, N. I. Muskhelishvili annv., Nauka, Moscow, 1972, pp. 203-207 